\title{
Lipschitz minimality of Hopf fibrations and Hopf vector fields
}

\author{
DENNIS DETURCK \\ HERMAN GLUCK \\ PETER STORM
}

Given a Hopf fibration of a round sphere by parallel great subspheres, we prove that the projection map to the base space is, up to isometries of domain and range, the unique Lipschitz constant minimizer in its homotopy class.

Similarly, given a Hopf fibration of a round sphere by parallel great circles, we view a unit vector field tangent to the fibers as a cross-section of the unit tangent bundle of the sphere, and prove that it is, up to isometries of domain and range, the unique Lipschitz constant minimizer in its homotopy class.

Previous attempts to find a mathematical sense in which Hopf fibrations and Hopf vector fields are optimal have met with limited success.

53C23, 53C30, 55R10, 55R25, 57R22, 57R25, 57R35; 53C 38, 53C43

\section{Prologue}

\section{Background}

The Hopf fibration $S^{3} \rightarrow S^{2}$ was the first example of a homotopically nontrivial map from a sphere to another sphere of lower dimension, and as such its discovery signaled the birth of homotopy theory. This map and its higher-dimensional cousins $S^{2 n+1} \rightarrow \mathbb{C P}^{n}, S^{4 n+1} \rightarrow \mathbb{H P}^{n}$ and $S^{15} \rightarrow S^{8}$-all of them fibrations of round spheres by great subspheres - are among the most strikingly beautiful maps in geometry and topology. And likewise for the Hopf vector fields on odd-dimensional spheres, that is, the unit vector fields on $S^{2 n+1}$ tangent to the great circle fibers of $S^{2 n+1} \rightarrow \mathbb{C P}^{n}$.

Yet in what mathematical sense are these objects optimal-what functional on the space of maps or vector fields do they minimize?

Fuller [8] and Eells and Lemaire [6] showed that Hopf projections are harmonic maps, but unfortunately harmonic maps from spheres to compact Riemannian manifolds are always unstable (see Xin [37]), and hence never energy-minimizing. Gluck and 
Ziller [16] used calibrations to show that the Hopf vector field on $S^{3}$, when viewed as a cycle inside the unit tangent bundle $U S^{3}$, is volume-minimizing in its homology class. But David Johnson [23] showed that this fails on the 5-sphere, and likewise on all higher odd-dimensional spheres.

\section{What we do here}

We find a mathematical sense in which Hopf fibrations and Hopf vector fields are optimal. We prove that Hopf fibrations are Lipschitz constant minimizers in their homotopy classes, uniquely so up to composition with isometries of domain and range. We do the same for Hopf vector fields, viewed as maps of spheres into their own unit tangent bundles.

\section{Where does this lead?}

We suspect that many natural geometric maps, such as Riemannian submersions of compact homogeneous spaces, are Lipschitz constant minimizers in their homotopy classes, unique up to composition with isometries of domain and range. The Hopf projections and Hopf vector fields are examples. We give one further example in this paper, and prove that the Stiefel projection of the space $V_{2} \mathbb{R}^{4}$ of orthonormal 2-frames in 4-space to the Grassmann manifold $G_{2} \mathbb{R}^{4}$ of oriented 2-planes through the origin in 4-space is a Lipschitz constant minimizer in its homotopy class, unique up to isometries of domain and range. In a forthcoming paper, Haomin Wen introduces new techniques to prove this for group multiplication $S^{3} \times S^{3} \rightarrow S^{3}$ on the round 3-sphere.

\section{Introduction and statement of results}

The Hopf fibration $S^{1} \subset S^{3} \rightarrow S^{2}$ of a round 3-sphere by parallel great circles was introduced by Heinz Hopf [20]. It provided the first example of a homotopically nontrivial map from one sphere to another of lower dimension, spurring the development of both homotopy theory and fiber spaces in their infancy. Although Hopf first presented his map in terms of quadratic polynomials, he explained later in this paper that the fibers are the intersections of $S^{3}$ with the complex lines through the origin in $\mathbb{R}^{4}=\mathbb{C}^{2}$. 
In his second paper on this theme [21], Hopf presented three families of fibrations of round spheres by parallel great subspheres:

$$
\begin{array}{lll}
S^{1} \subset S^{3} \rightarrow S^{2}=\mathbb{C P}^{1}, & S^{1} \subset S^{5} \rightarrow \mathbb{C P}^{2}, \ldots, & S^{1} \subset S^{2 n+1} \rightarrow \mathbb{C P}^{n}, \ldots \\
S^{3} \subset S^{7} \rightarrow S^{4}=\mathbb{H P}^{1}, & S^{3} \subset S^{11} \rightarrow \mathbb{H P}^{2}, \ldots, & S^{3} \subset S^{4 n+3} \rightarrow \mathbb{H P}^{n}, \ldots \\
S^{7} \subset S^{15} \rightarrow S^{8}, &
\end{array}
$$

with base spaces the complex and quaternionic projective spaces, and with the nonassociativity of the Cayley numbers responsible for the truncation of the third family.

This list is complete in the sense that any fibration of a round sphere by parallel great subspheres is isometric to one of the above (see Wong [34], Wolf [33; 32], Escobales [7], Ranjan [27]), meaning there is an isometry of the total space carrying fibers to fibers; see also Gluck, Warner and Ziller [14; 15]. A deeper uniqueness result, without the hypothesis that the fibers are great subspheres, is due to Gromoll and Grove $[17 ; 18]$ and Wilking [31].

The isometry groups of these Hopf fibrations act transitively on the spherical total spaces, and so the base spaces inherit from them Riemannian metrics which make the projection maps into Riemannian submersions. In particular, the spherical base spaces $S^{2}, S^{4}$ and $S^{8}$ all have radius $\frac{1}{2}$.

We begin now with Theorem 1 .

The Lipschitz constant Lip $f$ of a continuous map $f: X \rightarrow Y$ between metric spaces is the smallest number $c \geq 0$ such that $d\left(f(x), f\left(x^{\prime}\right)\right) \leq c d\left(x, x^{\prime}\right)$ for all points $x$ and $x^{\prime}$ in $X$. If no such number $c$ exists, we regard the Lipschitz constant of $f$ to be infinite. Since the above Hopf projections are Riemannian submersions, they all have Lipschitz constant 1.

Two maps $f_{1}$ and $f_{2}: X \rightarrow Y$ between metric spaces will be said to be isometric if there are isometries $g_{X}: X \rightarrow X$ and $g_{Y}: Y \rightarrow Y$ such that $g_{Y} f_{1}=f_{2} g_{X}$.

Theorem 1 The Lipschitz constant of any continuous map

$$
S^{2 n+1} \rightarrow \mathbb{C P}^{n} \quad \text { or } \quad S^{4 n+3} \rightarrow \mathbb{H P}^{n} \quad \text { or } \quad S^{15} \rightarrow S^{8}
$$

with nonzero Hopf invariant is greater than or equal to 1 and equals 1 if and only if the map is isometric to the corresponding Hopf projection.

In particular, the Hopf projections are, up to isometries of domain and range, the unique Lipschitz constant minimizers in their homotopy classes. 
The proof is entirely elementary metric geometry. Aiming for clarity of presentation, we give the argument first in the case of the original Hopf fibration $S^{1} \subset S^{3} \rightarrow S^{2}$, then in the case of $S^{1} \subset S^{5} \rightarrow \mathbb{C P}^{2}$, and after that explain the minor adjustments needed to carry out the proof in general.

We turn to Theorem 2.

Let $S^{n}$ denote the round $n$-sphere of radius 1 , let $S^{n} \times S^{n}$ be given the product metric, let $\Delta S^{n}=\left\{(x, x) \mid x \in S^{n}\right\} \subset S^{n} \times S^{n}$ be the diagonal, which is isometric to a round $n$-sphere of radius $\sqrt{2}$, and let $i: \Delta S^{n} \rightarrow S^{n} \times S^{n}$ denote the inclusion map.

Theorem 2 The Lipschitz constant of any map $\Delta S^{n} \rightarrow S^{n} \times S^{n}$ which is homotopic to the inclusion is greater than or equal to 1 and equals 1 if and only if the map is isometric to the inclusion.

This result can be appreciated by contrasting it with the following facts, pointed out to us some time ago by Walter Wei [29].

(1) The diagonal circle $i: \Delta S^{1} \rightarrow S^{1} \times S^{1}$ is length-minimizing in its homology class, and any other length-minimizer in that class is isometric to it.

(2) The diagonal 2-sphere $i: \Delta S^{2} \rightarrow S^{2} \times S^{2}$ is area-minimizing in its homology class, but there are other area-minimizers, such as $S^{2} \vee S^{2}$, in the same class.

(3) For $n \geq 3$, the diagonal $n$-sphere $i: \Delta S^{n} \rightarrow S^{n} \times S^{n}$ is not volume-minimizing in its homology class, since $S^{n} \vee S^{n}$ lies in the same class but has smaller volume.

Thus, minimizing the "stretch" (Lipschitz constant) of a map in its homotopy class may be viewed as an alternative to minimizing the area or volume of a cycle in its homology class, and yields different results.

We turn to Theorem 3.

Given a Hopf fibration of $S^{2 n+1}$ by parallel great circles, let $v$ denote either of the two unit vector fields on $S^{2 n+1}$ which are tangent to these fibers. Then define the mapping $V: S^{2 n+1} \rightarrow U S^{2 n+1}$ by $V(x)=(x, v(x))$, so that $V$ is the corresponding crosssection of the unit tangent bundle of $S^{2 n+1}$. We will also refer to the image $V\left(S^{2 n+1}\right)$ as a "Hopf vector field", and let $i: V\left(S^{2 n+1}\right) \rightarrow U S^{2 n+1}$ denote the inclusion map.

Theorem 3 The Lipschitz constant of any map of the Hopf vector field $V\left(S^{2 n+1}\right)$ into the unit tangent bundle $U S^{2 n+1}$ which is homotopic to the inclusion is greater than or equal to 1 and equals 1 if and only if the map is isometric to the inclusion. 
In other words, Hopf vector fields are, up to isometries of domain and range, the unique Lipschitz constant minimizers in their homotopy classes. This theorem, which asserts the Lipschitz minimality of Hopf vector fields on spheres, may be compared with attempts to prove their volume-minimality within the unit tangent bundle, as follows.

(1) On the 3-sphere, the 3-cycle $V\left(S^{3}\right) \subset U S^{3}$ is volume-minimizing in its homology class. This was shown by a calibrated geometry argument in Gluck and Ziller [16] .

(2) On the 5-sphere, the 5-cycle $V\left(S^{5}\right) \subset U S^{5}$ is not volume-minimizing in its homology class, and indeed, is not even a local minimum. This was shown by David Johnson [23], and likewise on all higher odd-dimensional spheres.

(3) Sharon Pedersen [26] showed that on each odd-dimensional sphere, beginning with $S^{5}$, there exist unit vector fields of exceptionally small volume which converge to a vector field with one singularity. She conjectured that on these spheres there are no unit vector fields of minimum volume at all, but that instead her limiting vector-field-with-singularity has minimum volume in its homology class in the unit tangent bundle. To support this, she showed that, as the ambient dimension increases, the volumes of her singular fields grow at the same rate as the known lower bound for volumes of nonsingular vector fields.

Remark - If the smooth submanifold $M$ of the Riemannian manifold $N$ is a volume-minimizing cycle in its homology class, then the inclusion map $i: M \rightarrow N$ is a Lipschitz constant minimizer in its homotopy class.

- Theorem 3 will follow quickly from Theorem 2.

We conclude with Theorem 4 .

We suspect that many natural geometric maps, such as Riemannian submersions of compact homogeneous spaces, are Lipschitz constant minimizers in their homotopy classes, unique up to isometries of domain and range.

We give one further example of this in the theorem below.

Let $V_{2} \mathbb{R}^{4}$ be the Stiefel manifold of orthonormal 2-frames in 4-space, with the metric inherited from its natural inclusion in $S^{3} \times S^{3}$, and let $G_{2} \mathbb{R}^{4}$ be the Grassmann manifold of oriented 2-planes through the origin in 4-space. The natural projection map $V_{2} \mathbb{R}^{4} \rightarrow G_{2} \mathbb{R}^{4}$ takes an orthonormal 2-frame to the 2-plane oriented by this ordered basis, and has Lipschitz constant 1 with respect to the Riemannian submersion metric that it induces on the Grassmann manifold. 
Theorem 4 The Lipschitz constant of any map of $V_{2} \mathbb{R}^{4} \rightarrow G_{2} \mathbb{R}^{4}$ homotopic to the Stiefel projection is greater than or equal to 1 with equality if and only if the map is isometric to this projection.

To prove this theorem, we will observe within the Stiefel projection $V_{2} \mathbb{R}^{4} \rightarrow G_{2} \mathbb{R}^{4}$ two families of Hopf projections $S^{3} \rightarrow S^{2}$, whose Lipschitz minimality, unique up to isometries of domain and range, was established in Theorem 1. They provide the framework for the proof.

\section{Harmonic maps}

We noted above that, beginning on $S^{5}$, Hopf vector fields are no longer volumeminimizing cycles in their homology classes in the unit tangent bundle. So it is natural to ask if they might be energy-minimizers there.

If $L: V \rightarrow W$ is a linear map between inner product spaces, its energy $\|L\|^{2}$ is defined to be the sum of the squares of the entries in a matrix for $L$ with respect to orthonormal bases for both $V$ and $W$, and is easily checked to be independent of such choices.

The energy of a smooth map $f: M \rightarrow N$ between Riemannian manifolds (with $M$ compact) is then defined by

$$
E(f)=\frac{1}{2} \int_{x \in M}\left\|d f_{x}\right\|^{2} d(\mathrm{vol}) .
$$

Such a map is harmonic if it is a critical point of the energy function, that is, if

$$
\left.\frac{d E\left(f_{t}\right)}{d t}\right|_{t=0}=\left.\frac{d}{d t}\right|_{t=0} \frac{1}{2} \int_{x \in M}\left\|d\left(f_{t}\right)_{x}\right\|^{2} d(\mathrm{vol})=0
$$

for all one-parameter families $\left\{f_{t}\right\}$ of maps from $M \rightarrow N$ with $f_{0}=f$.

Hopf projections are harmonic maps (see Fuller [8], Eells and Lemaire [6]); unfortunately, harmonic maps from spheres to compact Riemannian manifolds are always unstable (see Xin [37]).

If a vector field $V$ on a Riemannian manifold $M$ is regarded as a map of $M$ to its tangent bundle $T M$, then $V$ is harmonic if and only if it is parallel (see Nouhaud [25], Ishihara [22] and Konderak [24]).

By contrast, if a unit vector field on $M$ is regarded as a map into its unit tangent bundle $U M$ with the standard Sasaki metric, then Hopf vector fields $V_{H}$ on all odddimensional spheres are unstable harmonic maps. On $S^{3}$ there are no other unit vector fields which are harmonic (see Han and Yim [19]). 
If we now only look at cross-sections of the unit tangent bundle $U M$, rather than at all maps of $M \rightarrow U M$, then the Hopf vector fields $V_{H}: S^{n} \rightarrow U S^{n}$ are still unstable for $n=5,7,9, \ldots$ (see Wood [35]). But for $n=3$ they are stable, and in fact local minima of the energy (see Wood [36]).

The relation between volume and energy of unit vector fields on spheres and related spaces has been studied over the past decade by Olga Gil-Medrano and her collaborators. A cross-section of their papers is listed in the references (Borrelli, Brito and GilMedrano [1], Borrelli and Gil-Medrano [2], Brito [4], Brito and Walczak [5], GilMedrano [9; 10; 11] and Gil-Medrano and Llinares-Fuster [12]).

Acknowledgements We are especially grateful to Shmuel Weinberger, whose interest in determining the minimum Lipschitz constant $L(d)$ for maps from $S^{3} \rightarrow S^{2}$ of Hopf invariant $d$, and the asymptotic behavior of this function for large $d$, got us into this subject in the first place.

Many thanks to Dennis Sullivan for the proof of the Key Lemma used here in the argument for Theorem 2, and also to Rob Schneiderman and David Yetter for the main ideas in an earlier proof of this lemma, centered on immersions of $n$-manifolds in $2 n-$ space.

Special thanks to Olga Gil-Medrano, Paul Melvin, Shea Vela-Vick and Clayton Shonkwiler, as well as to Kerstin Baer, Eric Korman and Haomin Wen, for their substantial help during the preparation of this paper.

Storm was partially supported by NSF grant DMS-0904355 and the Roberta and Stanley Bogen Visiting Professorship at Hebrew University.

\section{Proof of Theorem 1 for maps from $S^{3}$ to $S^{2}$}

\section{Linking}

Since the Hopf invariant of a map reports linking of inverse images, we begin by commenting on this from two perspectives, homology and cohomology.

Homology Let $K$ and $K^{\prime}$ be disjoint oriented smooth simple closed curves in $\mathbb{R}^{3}$. Let $S$ and $S^{\prime}$ be oriented surfaces bounded by $K$ and $K^{\prime}$, in general position with respect to one another. Then the linking number $\operatorname{Lk}\left(K, K^{\prime}\right)$ of $K$ and $K^{\prime}$ can be defined to be the oriented intersection number of $K$ with $S^{\prime}$ or of $K^{\prime}$ with $S$, and standard arguments show that both quantities are equal, and hence independent of the choices of $S$ and $S^{\prime}$. 
Cohomology Given $K$ and $K^{\prime}$ as above, they have disjoint open tubular neighborhoods $U$ and $U^{\prime}$, each an open solid torus. By Poincaré duality, the one-dimensional homology of $U$ is isomorphic to its two-dimensional cohomology with compact support, $H_{1}(U ; \mathbb{Z}) \cong H_{c}^{2}(U ; \mathbb{Z})$, and likewise for $U^{\prime}$. Let $\beta$ and $\beta^{\prime}$ be 2 -forms with compact support in $U$ and $U^{\prime}$ which are dual in this way to $K$ and $K^{\prime}$.

Extend $\beta$ and $\beta^{\prime}$ over $\mathbb{R}^{3}$ to be zero outside $U$ and $U^{\prime}$, and then let $\alpha$ and $\alpha^{\prime}$ be 1 -forms with compact support in $\mathbb{R}^{3}$ such that $d \alpha=\beta$ and $d \alpha^{\prime}=\beta^{\prime}$. Then we can define

$$
\operatorname{Lk}\left(K, K^{\prime}\right)=\int_{\mathbb{R}^{3}} \alpha \wedge \beta^{\prime}=\int_{\mathbb{R}^{3}} \alpha^{\prime} \wedge \beta,
$$

and standard arguments show that both integrals are equal, hence independent of the choices of $\alpha$ and $\alpha^{\prime}$, and that this definition of linking number coincides with the one given above.

\section{The Hopf invariant of a map from $S^{3}$ to $S^{2}$}

We give here two equivalent definitions of the Hopf invariant of a continuous map $f: S^{3} \rightarrow S^{2}$ and refer the reader to Bott and Tu [3, pages 227-239] for further details.

(1) Homotope $f$ to a smooth map, which we still call $f$, and take any two regular values $y$ and $y^{\prime}$. Then the inverse images $f^{-1}(y)$ and $f^{-1}\left(y^{\prime}\right)$ are smooth 1-dimensional submanifolds of $S^{3}$, hence each is a finite union, say $K$ and $K^{\prime}$, of smooth simple closed curves, which we orient as follows. Start with orientations of the domain $S^{3}$ and the range $S^{2}$. Suppose $x$ is a point of $K=f^{-1}(y)$. Choose a small disk $D_{x}$ in $S^{3}$ through $x$, transverse there to $K$. Orient $D_{x}$ so that the restriction of $f$ to it is orientation-preserving. Then orient the component $K_{x}$ of $K$ containing $x$ so that the orientation of $D_{x}$ followed by the orientation of $K_{x}$ agrees with the orientation of $S^{3}$. Continue in this way to orient all the components of $K$ and $K^{\prime}$. Then define the Hopf invariant of $f$ to be the total linking number of all the components of $K$ with all the components of $K^{\prime}$.

Hopf [20] showed that this definition is independent of the choice of regular values $y$ and $y^{\prime}$ of $f$, and that it depends only on the homotopy class of $f$, not on the particular choice of $f$ itself.

(2) Use singular cohomology with integer coefficients, and let $\omega$ be a 2-dimensional cocycle on $S^{2}$ with $\left\langle\omega, S^{2}\right\rangle=1$. Then the pullback $f^{*} \omega$ is a 2-cocycle on $S^{3}$. Since $H^{2}\left(S^{3} ; \mathbb{Z}\right)=0$, there is a 1 -dimensional integral cochain $\alpha$ on $S^{3}$ such that $d \alpha=f^{*} \alpha$. Then the integer $\left\langle\alpha \cup f^{*} \omega, S^{3}\right\rangle$ is defined to be the Hopf invariant of $f$. Note that we are using " $d$ " instead of " $\delta$ " for the coboundary map, as in the case of differential forms. 
One shows that this definition is independent of the choice of 2-cocycle $\omega$ on $S^{2}$, and of the choice of 1 -cochain $\alpha$ on $S^{3}$ such that $d \alpha=f^{*} \omega$, and that it depends only on the homotopy class of $f$, not on the particular choice of $f$ itself.

Unlike Hopf's definition, this one does not require us to first homotope $f$ to make it smooth. However, if $f$ is smooth, we can use de Rham cohomology, let $\omega$ be a smooth 2-form on $S^{2}$ such that $\int_{S^{2}} \omega=1$, let $\alpha$ be a smooth 1 -form on $S^{3}$ such that $d \alpha=f^{*} \omega$, and then the integral $\int_{S^{3}} \alpha \wedge f^{*} \omega$ gives the Hopf invariant of $f$. This is the approach of J H C Whithead [30], who showed it to be equivalent to Hopf's.

\section{Mix-and-match formula for the Hopf invariant}

Since we will be looking at all continuous maps $f: S^{3} \rightarrow S^{2}$, not known in advance to be smooth, we favor Whitehead's approach to the Hopf invariant, phrased as above in the language of singular cohomology with integer coefficients.

Here is a curiosity of that approach. Initially it is just a play with two actors: the 2-dimensional cocycle $\omega$ on $S^{2}$ with $\left\langle\omega, S^{2}\right\rangle=1$, and the 1-dimensional cochain $\alpha$ on $S^{3}$ with $d \alpha=f^{*} \omega$, with the Hopf invariant of $f$ given by

$$
\operatorname{Hopf}(f)=\left\langle\alpha \cup f^{*} \omega, S^{3}\right\rangle .
$$

A third actor can be introduced: another 2-dimensional cocycle $\omega^{\prime}$ on $S^{2}$ with $\left\langle\omega^{\prime}, S^{2}\right\rangle=1$, and then we claim that

$$
\operatorname{Hopf}(f)=\left\langle\alpha \cup f^{*} \omega^{\prime}, S^{3}\right\rangle,
$$

which we view as a "mix-and-match" formula. To verify its correctness, note that $\omega$ and $\omega^{\prime}$ are cohomologous on $S^{2}$, so we can write $\omega-\omega^{\prime}=d \eta$, for some 1-cochain $\eta$ on $S^{2}$. Then

$$
\alpha \cup f^{*} \omega-\alpha \cup f^{*} \omega^{\prime}=\alpha \cup f^{*} d \eta=\alpha \cup d f^{*} \eta .
$$

Now

$$
d\left(\alpha \cup f^{*} \eta\right)=d \alpha \cup f^{*} \eta-\alpha \cup d f^{*} \eta .
$$

So integration by parts yields

$$
\begin{aligned}
\left\langle\alpha \cup f^{*} \omega, S^{3}\right\rangle-\left\langle\alpha \cup f^{*} \omega^{\prime}, S^{3}\right\rangle & =\left\langle\alpha \cup d f^{*} \eta, S^{3}\right\rangle \\
& =\left\langle d \alpha \cup f^{*} \eta, S^{3}\right\rangle-\left\langle d\left(\alpha \cup d^{*} \eta\right), S^{3}\right\rangle \\
& =\left\langle d \alpha \cup f^{*} \eta, S^{3}\right\rangle,
\end{aligned}
$$

since $\left\langle d\left(\alpha \cup f^{*} \eta\right), S^{3}\right\rangle=0$ by Stokes's theorem. 
But $d \alpha=f^{*} \omega$, and hence

$$
d \alpha \cup f^{*} \eta=f^{*} \omega \cup f^{*} \eta=f^{*}(\omega \cup \eta)=0,
$$

since $\omega \cup \eta$ is a 3 -form on $S^{2}$, and hence identically zero. This verifies the mix-andmatch formula above.

\section{A sufficient condition for the Hopf invariant to be zero}

We put the mix-and-match formula to immediate good use.

As motivation, suppose that $f: S^{3} \rightarrow S^{2}$ is a smooth map, with $y$ and $y^{\prime}$ as regular values, so that the Hopf invariant of $f$ is given by the formula

$$
\operatorname{Hopf}(f)=\operatorname{Lk}\left(K, K^{\prime}\right)
$$

where $K=f^{-1}(y)$ and $K^{\prime}=f^{-1}\left(y^{\prime}\right)$ are smooth oriented links in $S^{3}$.

Suppose there is an open set $U$ in $S^{3}$ which contains $K$, excludes $K^{\prime}$, and has trivial 1-dimensional homology:

$$
K \subset U \subset S^{3}-K^{\prime}, \quad H_{1}(U ; \mathbb{Z})=0 .
$$

Then the link $K$ bounds a 2-chain $S$ in $U$, automatically disjoint from $K^{\prime}$, and hence the linking number of $K$ and $K^{\prime}$ must be zero. Thus $\operatorname{Hopf}(f)=0$.

The following version of this, which applies to continuous rather than smooth maps, is suitable for our purposes.

Lemma 5 (Preliminary version) Let $f: S^{3} \rightarrow S^{2}$ be a continuous map, and let $y$ and $y^{\prime}$ be two points of $S^{2}$, with inverse images $K=f^{-1}(y)$ and $K^{\prime}=f^{-1}\left(y^{\prime}\right)$. Suppose there is an open set $U$ in $S^{3}$ such that

$$
K \subset U \subset \bar{U} \subset S^{3}-K^{\prime}, \quad H_{1}(U ; \mathbb{Z})=0 .
$$

Then the Hopf invariant of $f$ is zero.

Comment In the above statement, the symbol $\bar{U}$ denotes the closure of $U$, and if the chain of inclusions holds, we will say that $U$ separates $K$ from $K^{\prime}$. Note that if $U$ separates $K$ from $K^{\prime}$, then $S^{3}-\bar{U}$ separates $K^{\prime}$ from $K$.

Proof First we need to refine the above chain of inclusions by finding small open sets $V$ and $V^{\prime}$ about $y$ and $y^{\prime}$ in $S^{2}$ so that

$$
f^{-1}(V) \subset U \subset S^{3}-f^{-1}\left(V^{\prime}\right) .
$$


To find $V$, note that the image under $f$ of the compact set $S^{3}-U$ is compact and hence closed in $S^{2}$, and misses the point $y$ because $f^{-1}(y) \subset U$. Therefore $V=S^{2}-f\left(S^{3}-U\right)$ is an open neighborhood of $y$ in $S^{2}$ whose inverse image $f^{-1}(V)$ lies in $U$, as desired. To find $V^{\prime}$, repeat this with $S^{3}-\bar{U}$ in place of $U$ and $y^{\prime}$ in place of $y$.
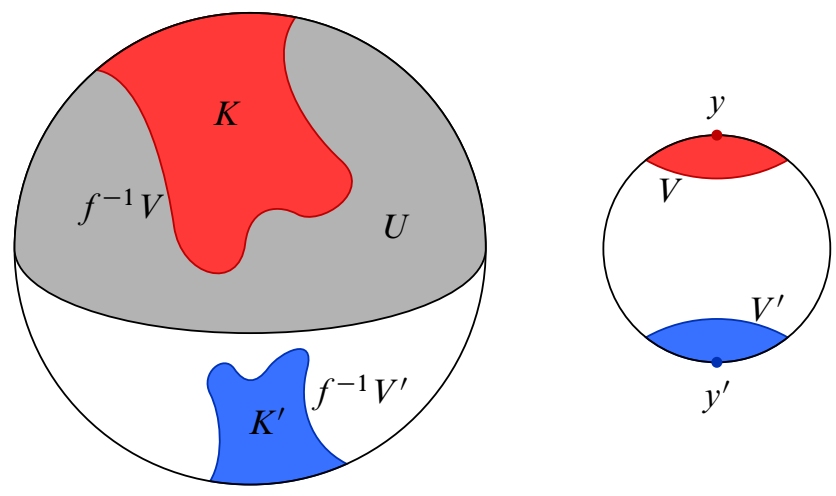

Figure 1: $f: S^{3} \rightarrow S^{2}$

Now let $\omega$ be a 2-dimensional singular cocycle on $S^{2}$ with support in $V$, such that $\left\langle\omega, S^{2}\right\rangle=1$, and likewise for $\omega^{\prime}$ and $V^{\prime}$.

Then $f^{*} \omega$ is a 2-dimensional singular cocycle on $S^{3}$ with support in $f^{-1}(V) \subset U$. By Poincare duality, the 2-dimensional singular cohomology of $U$ with compact supports, $H_{c}^{2}(U ; \mathbb{Z})$, is isomorphic to $H_{1}(U ; \mathbb{Z})$, which by hypothesis is zero. Hence there is a 1 -dimensional cochain $\alpha$ on $S^{3}$ with compact support inside $U$, such that $d \alpha=f^{*} \omega$.

Now $\alpha$ and $f^{*} \omega^{\prime}$ have supports inside the disjoint open sets $U$ and $f^{-1}\left(V^{\prime}\right)$, and therefore the cohomology class $\left[\alpha \cup f^{*} \omega^{\prime}\right]=0$. Then by the mix-and-match formula for the Hopf invariant, we have

$$
\operatorname{Hopf}(f)=\left\langle\alpha \cup f^{*} \omega^{\prime}, S^{3}\right\rangle=0 .
$$

\section{Plan of the proof of Theorem 1 for maps of $S^{3}$ to $S^{2}(1 / 2)$}

We will show that any continuous map $f: S^{3} \rightarrow S^{2}(1 / 2)$ with nonzero Hopf invariant has Lipschitz constant greater than or equal to 1 , with equality if and only if the map is isometric to the Hopf projection. There are four steps to the proof, as follows.

Step 1 We show that for each point $y \in S^{2}$, its inverse image $f^{-1}(y)$ lies on some great 2-sphere in $S^{3}$. 
Step 2 We show that each inverse image $f^{-1}(y)$ is a great circle in $S^{3}$.

Step 3 We show that any two such great circles $f^{-1}(y)$ and $f^{-1}\left(y^{\prime}\right)$ are parallel to one another.

Step 4 We conclude that $f$ is isometric to the Hopf projection.

In what follows, we use the phrases "fiber of $f$ " and "point-inverse-image of $f$ " interchangeably.

Step 1: Each fiber of $f$ lies on a great 2-sphere in $S^{3}$ Let $f: S^{3} \rightarrow S^{2}(1 / 2)$ be a map with nonzero Hopf invariant and with Lipschitz constant less than or equal to 1 . If $A$ is a subset of $S^{3}$ and $r$ is a positive real number, $N(A, r)$ will denote the open $r$-neighborhood of $A$,

$$
N(A, r)=\left\{p \in S^{3} \mid d(p, A)<r\right\} .
$$

We begin the argument by choosing at random a point $y \in S^{2}(1 / 2)$, and letting $K=f^{-1}(y)$ denote its inverse image in $S^{3}$. Since Lip $f \leq 1$, no point in $N(K, \pi / 2)$ can map to the antipodal point $-y$ in $S^{2}(1 / 2)$.

On the other hand, some point in $S^{3}$ must map to $-y$ because $f$ is homotopically nontrivial, and hence onto. Say $f(-x)=-y$.

Since Lip $f \leq 1$, the point $-x$ can not lie in $N(K, \pi / 2)$, and therefore no point of $K$ can lie in the open hemisphere $N(-x, \pi / 2)$. Hence $K$ must lie in the closed hemisphere of $S^{3}$ centered at $x$, as shown below.

We depict $y$ and $-y$ as north and south poles of $S^{2}(1 / 2)$, and $x$ and $-x$ as north and south poles of $S^{3}$, with $E S$ as the corresponding equatorial great 2-sphere.

Figure 2 shows $x$ lying outside $N(K, \pi / 2)$, and we argue now that this is correct.

Suppose to the contrary that $x$ lies inside $N(K, \pi / 2)$. Since $K$ lies in the closed northern hemisphere of $S^{3}$ centered at $x$, we know the half open geodesic arc $[p, x)$ from each point $p$ of $K$, up to but not including $x$, must lie in $N(K, \pi / 2)$. If $x$ also lies in $N(K, \pi / 2)$, then each closed geodesic arc $[p, x]$ lies in $N(K, \pi / 2)$.

Thus $K$ can be contracted along these geodesic arcs within $N(K, \pi / 2)$ to the single point $x$. If $f$ were smooth with regular values at $y$ and $-y$, this would be enough to show that the linking number of $K=f^{-1}(y)$ and $K^{\prime}=f^{-1}(-y)$ is zero, and hence that the Hopf invariant of $f$ is zero. This contradiction would then show that $x$ must indeed lie outside $N(K, \pi / 2)$, confirming the accuracy of the above figure.

But we don't know in advance that $f$ is smooth, and so must work a little harder to expose the contradiction. 


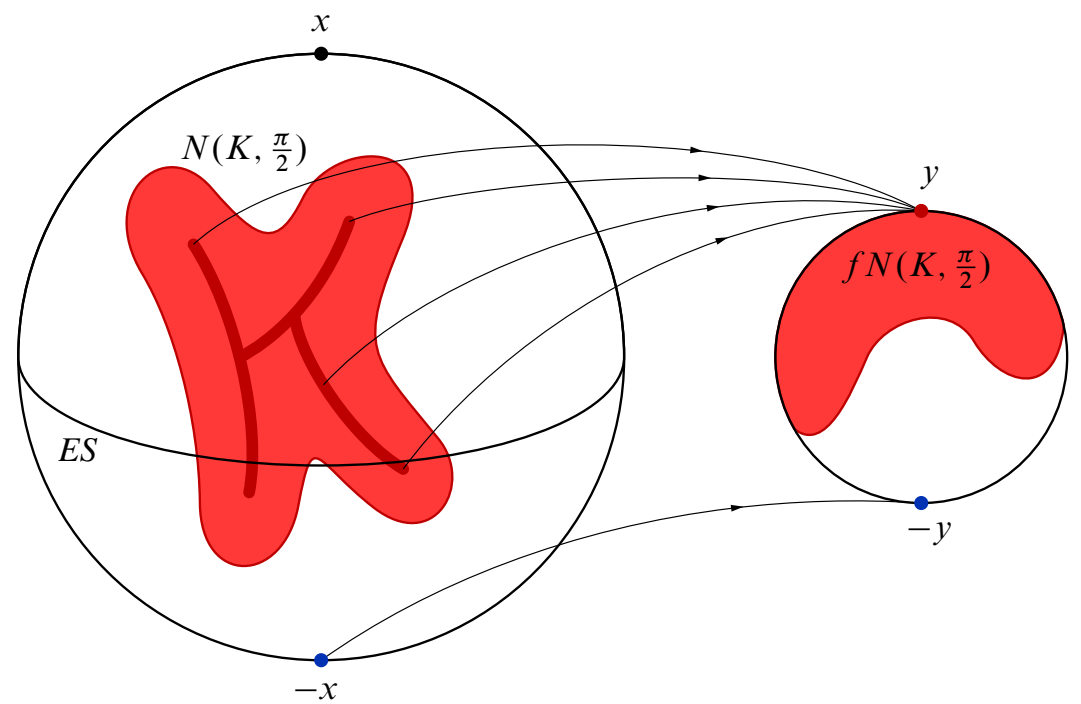

Figure 2: $f: S^{3} \rightarrow S^{2}\left(\frac{1}{2}\right)$

Consider our assumption (contrary to fact) that $x$ lies in the open set $N(K, \pi / 2)$. Then for some small $\varepsilon>0$, the closure of the $3 \varepsilon$-ball $N(x, 3 \varepsilon)$ also lies in $N(K, \pi / 2)$. It follows that

(1) the closure of the $2 \varepsilon$-ball $N(x, 2 \varepsilon)$ lies in $N(K, \pi / 2-\varepsilon)$.

Letting $K^{\prime}=f^{-1}(-y)$, and noting our assumption that Lip $f \leq 1$, we have

(2) $N(K, \pi / 2-\varepsilon)$ and $N\left(K^{\prime}, \varepsilon\right)$ must be disjoint.

Now let $C$ denote the cone over the open set $N(K, \varepsilon)$ from the north pole $x$ of $S$, meaning the union of all geodesic arcs from points of $N(K, \varepsilon)$ to $x$. Denote such a geodesic arc by $[p, x]$, and note that it has length less than $\pi / 2+\varepsilon$. If we stop that geodesic arc $2 \varepsilon$ short of $x$, say at the point $x^{\prime}$, then the subarc $\left[p, x^{\prime}\right]$ lies entirely in $N(K, \pi / 2-\varepsilon)$.

We can complete the trip along the geodesic arc from $x^{\prime}$ to $x$ within the closure of the ball $N(x, 2 \varepsilon)$, and hence by (1) above within the open set $N(K, \pi / 2-\varepsilon)$.

Now let

$$
U=C \cup N(x, 2 \varepsilon),
$$

the union of two cones in $S^{3}$ with vertices at $x$. Since $C$ is a cone over the open set $N(K, \varepsilon)$, it is open at all of its points, save possibly at $x$. Addition of the open set $N(x, 2 \varepsilon)$ repairs this deficit, and so the set $U$ is open. As the union of two cones, it is contractible within itself to $x$. 


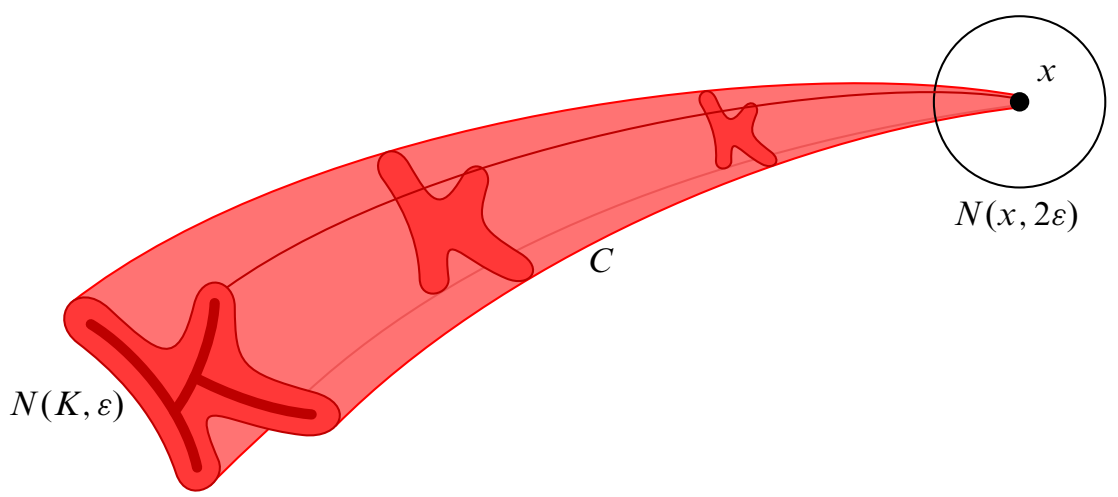

Figure 3: The contractible open set $U=C \cup N(x, 2 \varepsilon)$

By construction, we have

$$
K \subset U \subset N(K, \pi / 2-\varepsilon),
$$

which is disjoint from $N\left(K^{\prime}, \varepsilon\right)$ by (2) above. Hence

$$
K \subset U \subset \bar{U} \subset S^{3}-K^{\prime} .
$$

Since $U$ is contractible, it certainly satisfies $H_{1}(U ; \mathbb{Z})=0$.

Thus the conditions of Lemma 5 are satisfied, and we conclude that the Hopf invariant of $f$ is zero.

This contradiction shows that the north pole $x$ can not lie inside $N(K, \pi / 2)$, and confirms the accuracy of its placement in Figure 2.

Since $x$ cannot lie inside $N(K, \pi / 2)$, it follows that no point of $K$ can lie in the open northern hemisphere $N(x, \pi / 2)$. Since we already know that $K$ lies in the closed northern hemisphere, it follows that $K$ must lie on its boundary, the equatorial 2-sphere ES, completing Step 1.

Step 2: Each fiber of $\boldsymbol{f}$ is a great circle in $\boldsymbol{S}^{3}$ So far, we know that the fiber $K=f^{-1}(y)$ lies on the equatorial 2-sphere $E S$, and we intend to recreate there the same situation we had on the full 3-sphere $S^{3}$.

To begin, some point of the fiber $K^{\prime}=f^{-1}(-y)$ must also lie on $E S$. Otherwise, for a sufficiently small positive value of $\varepsilon$, the open equatorial region $U=N(E S, \varepsilon)$ would separate $K$ from $K^{\prime}$. Since $H_{1}(U ; \mathbb{Z})=0$, Lemma 5 would imply that $\operatorname{Hopf}(f)=0$. 
Stealing notation from the previous section, let $-x$ now denote a point of $E S$ with $f(-x)=-y$, so that $-x$ lies in the fiber $K^{\prime}=f^{-1}(-y)$. Note that this point $-x$ is entirely different from the point of the same name in the previous section.

Since Lip $f \leq 1$, the point $-x$ can not lie in $N(K, \pi / 2)$, and therefore no point of $K$ can lie in the open 2-dimensional hemisphere $\operatorname{ES} \cap N(-x, \pi / 2)$. Hence $K$ must lie in the closed hemisphere of $E S$ centered at $x$.

If the point $x$ were to lie inside $N(K, \pi / 2)$, then, just as in the previous section, we would find a contractible open subset $U$ of $S^{3}$ which separates $K$ from $K^{\prime}$, which once again by Lemma 5 would imply that $\operatorname{Hopf}(f)=0$.

Thus $x$ cannot lie inside $N(K, \pi / 2)$, and it follows that no point of $K$ can lie in the open hemisphere of $E S$ centered at $x$. Since $K$ lies in that closed hemisphere, it must in fact lie on its boundary great circle $E C$.

We now assert that $K$ can not be a proper subset of $E C$, and see this in three cases as follows, supported by Figure 4 below.

Assume for the moment that $K$ is a proper subset of the great circle $E C$.

Case 1: $\boldsymbol{K}^{\prime}$ is disjoint from $\boldsymbol{E} \boldsymbol{C}$ Then an open 3-cell $U$ as shown in Figure 4 separates $K$ from $K^{\prime}$.
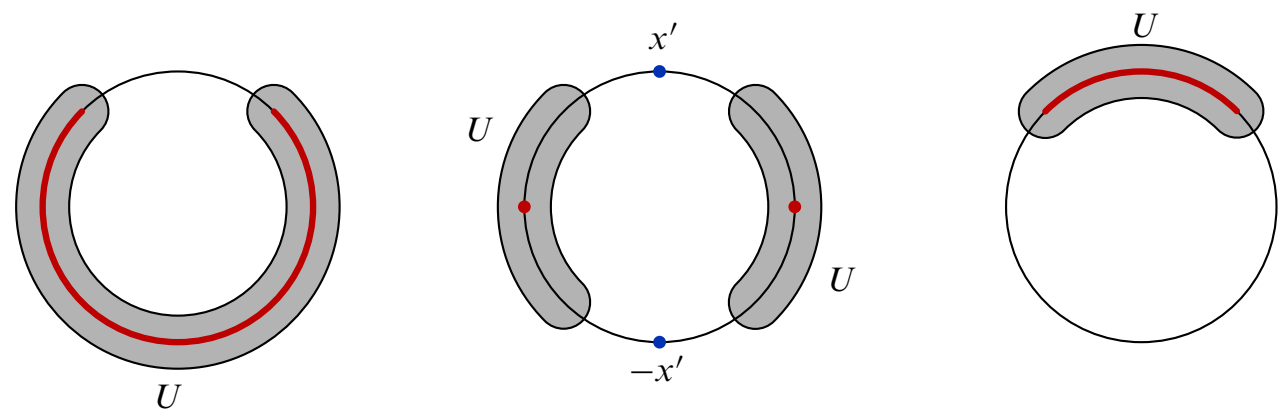

Figure 4: The fiber $K$ can not be a proper subset of the great circle $E C$.

Case 2: $\boldsymbol{K}^{\prime}$ meets $\boldsymbol{E} \boldsymbol{C}$ in two antipodal points $\boldsymbol{x}^{\prime}$ and $-\boldsymbol{x}^{\prime}$ Then each of $x^{\prime}$ and $-x^{\prime}$ sits at the center of an open semicircle on $E C$ which is forbidden to contain any points of $K$, since the distance in $S^{3}$ between $K$ and $K^{\prime}$ is $\geq \pi / 2$. So $K$ consists at most of two points, and then the disjoint union $U$ of two open three-cells, as shown in the figure, separates $K$ from $K^{\prime}$.

Case 3: $\boldsymbol{K}^{\prime}$ meets $\boldsymbol{E} \boldsymbol{C}$, but not just in two antipodal points Then, as in Case 2 above, $K$ is forbidden to lie in a union of open semicircles on $E C$, which in the present 
case is an open arc on $E C$. Therefore $K$ is constrained to lie in the complementary closed arc, and then the open 3-cell $U$ shown in the figure separates $K$ from $K^{\prime}$.

In each of the three cases above we have $H_{1}(U ; \mathbb{Z})=0$, and then Lemma 5 would imply that $\operatorname{Hopf}(f)=0$.

This contradiction shows that $K=f^{-1}(y)$ must be the entire great circle $E C$.

Since $y$ was an arbitrary point of $S^{2}$, we now know that all the fibers of $f$ are great circles in $S^{3}$.

Step 3: Any two great circle fibers of $\boldsymbol{f}$ are parallel to one another We claim now that any two great circle fibers of $f$ are parallel, meaning that they are a constant distance apart from one another, and see this as follows.

Refer again to any pair of antipodal points $y$ and $-y$ on $S^{2}(1 / 2)$, and to their inverse images $K=f^{-1}(y)$ and $K^{\prime}=f^{-1}(-y)$ in $S^{3}$, now known to be great circles.

No point of $K$ can be closer than $\pi / 2$ to any point of $K^{\prime}$, since their images $y$ and $-y$ under $f$ are exactly $\pi / 2$ apart on $S^{2}(1 / 2)$ and we have $\operatorname{Lip} f \leq 1$.

Thus the great circles $K$ and $K^{\prime}$ on $S^{3}$ are orthogonal, meaning that they are the unit circles on a pair of orthogonal 2-planes through the origin in $\mathbb{R}^{4}$.

Now let $z$ be a point on $S^{2}(1 / 2)$ of distance $\alpha$ from $y$ and distance $\pi / 2-\alpha$ from $-y$.

Let $K^{\prime \prime}=f^{-1}(z)$ be the corresponding great circle fiber. Where does $K^{\prime \prime}$ lie in $S^{3}$ with reference to $K$ and $K^{\prime}$ ?

To answer that, consider the tubular neighborhoods $N(K, \alpha)$ and $N\left(K^{\prime}, \pi / 2-\alpha\right)$ about $K$ and $K^{\prime}$ in $S^{3}$. Each is an open solid torus, and their common boundary, call it $T_{\alpha}$, is a 2 -dimensional torus, as shown in the figure below.

We claim that the great circle fiber $K^{\prime \prime}=f^{-1}(z)$ must lie entirely on the 2-dimensional torus $T_{\alpha}$.

If a part of $K^{\prime \prime}$ intrudes into the open set $N(K, \alpha)$, then that part is closer than $\alpha$ to $K$, yet is mapped by $f$ to the point $z$ which is exactly at distance $\alpha$ from $f(K)=y$, in contradiction to the assumption that $\operatorname{Lip} f \leq 1$.

We get a similar contradiction if $K^{\prime \prime}$ intrudes into the open set $N\left(K^{\prime}, \pi / 2-\alpha\right)$.

Hence $K^{\prime \prime}$ lies on the common boundary $T_{\alpha}$ of these two open sets, and so is at constant distance $\alpha$ from $K$ and at constant distance $\pi / 2-\alpha$ from $K^{\prime}$.

Since $y$ and $z$ were arbitrary nonantipodal points on $S^{2}(1 / 2)$, we conclude that all the great circle fibers of the map $f$ are parallel to one another, as claimed. 


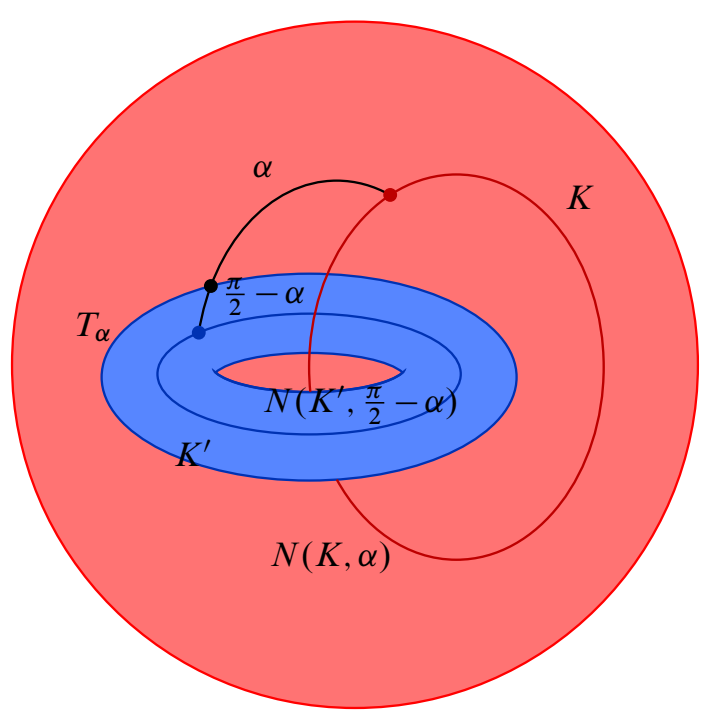

Figure 5: $S^{3}=N(K, \alpha) \cup T_{\alpha} \cup N\left(K^{\prime}, \frac{\pi}{2}-\alpha\right)$

Step 4. The map $\boldsymbol{f}$ is isometric to the Hopf projection We have been considering a homotopically nontrivial map $f: S^{3} \rightarrow S^{2}(1 / 2)$ with Lipschitz constant less than or equal to 1 , and have so far shown that the fibers of $f$ are parallel great circles on $S^{3}$. But, as mentioned earlier, any fibration of $S^{3}$ by parallel great circles is isometric to the Hopf fibration. So, $f$ induces a homotopically nontrivial map $\bar{f}: S^{2}(1 / 2) \rightarrow S^{2}(1 / 2)$ with Lipschitz constant less than or equal to 1 , and this is easily seen to be an isometry. It follows that the map $f$ must be isometric to the Hopf projection.

This completes the proof of Theorem 1 in this first instance, and displays the style of argument that we will emulate for the general case.

\section{Proof of Theorem 1 for all Hopf projections}

\section{The Hopf invariant}

Look once again at our display of all the Hopf fibrations of round spheres by parallel great subspheres:

$$
\begin{array}{lll}
S^{1} \subset S^{3} \rightarrow S^{2}=\mathbb{C P}^{1}, & S^{1} \subset S^{5} \rightarrow \mathbb{C P}^{2}, \ldots, & S^{1} \subset S^{2 n+1} \rightarrow \mathbb{C P}^{n}, \ldots \\
S^{3} \subset S^{7} \rightarrow S^{4}=\mathbb{H P}^{1}, & S^{3} \subset S^{11} \rightarrow \mathbb{H P}^{2}, \ldots, & S^{3} \subset S^{4 n+3} \rightarrow \mathbb{H P}^{n}, \ldots \\
S^{7} \subset S^{15} \rightarrow S^{8} &
\end{array}
$$


We suppose that $f$ is a continuous map from

$$
S^{2 n+1} \rightarrow \mathbb{C P}^{n}, \quad S^{4 n+1} \rightarrow \mathbb{H P}^{n}, \quad \text { or } \quad S^{15} \rightarrow S^{8},
$$

and intend to give two equivalent definitions of its Hopf invariant.

Before doing that, we recall the topology of the base spaces.

A choice of ascending complex vector spaces $\mathbb{C}^{1} \subset \mathbb{C}^{2} \subset \mathbb{C}^{3} \subset \cdots$ leads to an ascending sequence of complex projective spaces $\mathbb{C P}^{0} \subset \mathbb{C P}^{1} \subset \mathbb{C P}^{2} \subset \cdots$. The cohomology ring $H^{*}\left(\mathbb{C P}^{n} ; \mathbb{Z}\right)$ is a truncated polynomial ring with one generator $[\omega]$ in dimension 2 and with the relation $[\omega]^{n+1}=0$. We can take $\omega$ to be the Kähler form, scaled so that it integrates to 1 over $\mathbb{C P}^{1}=S^{2}(1 / 2)$.

A choice of ascending quaternionic vector spaces $\mathbb{H}^{1} \subset \mathbb{H}^{2} \subset \mathbb{H}^{3} \subset \cdots$ leads to an ascending sequence of quaternionic projective spaces $\mathbb{H P}^{0} \subset \mathbb{H P}^{1} \subset \mathbb{H P}^{2} \subset \cdots$. The cohomology ring $H^{*}\left(\mathbb{H} \mathbb{P}^{n} ; \mathbb{Z}\right)$ is a truncated polynomial ring with one generator $[\omega]$ in dimension 4 and with the relation $[\omega]^{n+1}=0$. We can take $\omega$ to be the quaternionic Kähler form, scaled so that it integrates to 1 over $\mathbb{H P}^{1}=S^{4}(1 / 2)$.

For simplicity of expression and to gain the advantage of making our arguments more concrete, we will focus on maps $f: S^{2 n+1} \rightarrow \mathbb{C P}^{n}$, and then comment afterwards on the very slight changes needed to handle maps of $S^{4 n+1} \rightarrow \mathbb{H} \mathbb{P}^{n}$ and of $S^{15} \rightarrow S^{8}$.

(1) Given a map $f: S^{2 n+1} \rightarrow \mathbb{C P}^{n}$, homotope it so that it is smooth, has a given value $y$ in $\mathbb{C P}^{n}$ as regular value, and so that it is transverse to the corresponding "antipodal" $\mathbb{C P}^{n-1}$, which is simply the cut locus of $y$ in the usual Riemannian metric on $\mathbb{C} \mathbb{P}^{n}$. Then the inverse image $K=f^{-1}(y)$ is a finite union of smooth simple closed curves in $S^{2 n+1}$, while the inverse image $K^{\prime}=f^{-1}\left(\mathbb{C P}^{n-1}\right)$ is a smooth submanifold of $S^{2 n+1}$ of dimension $2 n-1$.

Orienting $S^{2 n+1}$ arbitrarily, and $\mathbb{C} \mathbb{P}^{n}$ in the usual way, we derive orientations for $K$ and $K^{\prime}$ just as we did for maps of $S^{3} \rightarrow S^{2}$. Then the Hopf invariant of $f$ is defined to be the total linking number of all the components of $K$ with all the components of $K^{\prime}$.

This definition is independent of the choice of $y$ and $\mathbb{C P}^{n-1}$ in $\mathbb{C P}^{n}$, and depends only on the homotopy class of $f$.

(2) Let $\omega$ be the Kähler form on $\mathbb{C P}^{n}$, scaled so that $\left\langle\omega, \mathbb{C P}^{1}\right\rangle=1$. Then the pullback $f^{*} \omega$ is a $2-$ cocycle on $S^{2 n+1}$. Since $H^{2}\left(S^{2 n+1} ; \mathbb{Z}\right)=0$, there is a $1-$ dimensional integral cochain $\alpha$ on $S^{2 n+1}$ such that $d \alpha=f^{*} \omega$. Then the integer $\left\langle\alpha \cup\left(f^{*} \omega\right)^{n}, S^{2 n+1}\right\rangle$ is defined to be the Hopf invariant of $f$. 
One shows that this definition is independent of the choice of 2-cocycle $\omega$ on $\mathbb{C P}^{n}$ which generates $H^{2}\left(\mathbb{C P}^{n} ; \mathbb{Z}\right) \cong \mathbb{Z}$, and of the choice of 1 -cochain $\alpha$ on $S^{2 n+1}$ such that $d \alpha=f^{*} \omega$, and that it depends only on the homotopy class of $f$.

If $\mathrm{f}$ is smooth, we can use de Rham cohomology for this approach, just as we did for maps of $S^{3} \rightarrow S^{2}$.

\section{Mix-and-match formula for the Hopf invariant}

The situation here is the same as for maps of $S^{3} \rightarrow S^{2}$.

Suppose $\omega^{\prime}$ is another 2 -cocycle on $\mathbb{C P}^{n}$ with $\left\langle\omega^{\prime}, \mathbb{C P}^{1}\right\rangle=1$.

Then instead of the above formula,

$$
\operatorname{Hopf}(f)=\left\langle\alpha \cup\left(f^{*} \omega\right)^{n}, S^{2 n+1}\right\rangle,
$$

for the Hopf invariant, we have the mix-and-match formula,

$$
\operatorname{Hopf}(f)=\left\langle\alpha \cup\left(f^{*} \omega^{\prime}\right)^{n}, S^{2 n+1}\right\rangle .
$$

To verify this, first write $\omega-\omega^{\prime}=d \eta$ for some 1 -cochain $\eta$ on $\mathbb{C P}^{n}$.

It follows that $\omega^{n}-\left(\omega^{\prime}\right)^{n}=d \zeta$ for some $(2 n-1)-$ cochain $\zeta$ on $\mathbb{C P}^{n}$.

Write

$$
\alpha \cup\left(f^{*} \omega\right)^{n}-\alpha \cup\left(f^{*} \omega^{\prime}\right)^{n}=\alpha \cup f^{*} d \zeta=\alpha \cup d f^{*} \zeta,
$$

and then the integration by parts given earlier in the case of $S^{3} \rightarrow S^{2}$, now with $\zeta$ in place of $\eta$ there, finishes the present argument and confirms the mix-and-match formula above.

\section{A sufficient condition for the Hopf invariant to be zero}

Lemma 5 (Complete version) (1) Let $f: S^{2 n+1} \rightarrow \mathbb{C P}^{n}$ be a continuous map, and let $y$ and $\mathbb{C P}^{n-1}$ be a point and disjoint projective hyperplane in $\mathbb{C P}^{n}$, with inverse images $K=f^{-1}(y)$ and $K^{\prime}=f^{-1}\left(\mathbb{C P}^{n-1}\right)$. Suppose there is an open set $U$ in $S^{2 n+1}$ such that

$$
K \subset U \subset \bar{U} \subset S^{2 n+1}-K^{\prime} \text { and } H_{1}(U ; \mathbb{Z})=0 .
$$

Then the Hopf invariant of $f$ is zero.

(2) Let $f: S^{4 n+1} \rightarrow \mathbb{H P}^{n}$ be a continuous map, and let $y$ and $\mathbb{H P}^{n-1}$ be a point and disjoint projective hyperplane in $\mathbb{H P}^{n}$, with inverse images $K=f^{-1}(y)$ and $K^{\prime}=f^{-1}\left(\mathbb{H P}^{n-1}\right)$. Suppose there is an open set $U$ in $S^{4 n+1}$ such that

$$
K \subset U \subset \bar{U} \subset S^{4 n+1}-K^{\prime} \text { and } H_{3}(U ; \mathbb{Z})=0 .
$$

Then the Hopf invariant of $f$ is zero. 
(3) Let $f: S^{15} \rightarrow S^{8}$ be a continuous map, and let $y$ and $y^{\prime}$ be two points of $S^{8}$, with inverse images $K=f^{-1}(y)$ and $K^{\prime}=f^{-1}\left(y^{\prime}\right)$. Suppose there is an open set $U$ in $S^{15}$ such that

$$
K \subset U \subset \bar{U} \subset S^{15}-K^{\prime} \text { and } H_{7}(U ; \mathbb{Z})=0 .
$$

Then the Hopf invariant of $f$ is zero.

The proof is the same as for the prototype discussed earlier.

\section{Beginning the proof of Theorem 1}

We will give the proof for maps of $S^{5} \rightarrow \mathbb{C P}^{2}$, leaning heavily on the techniques developed for the case $S^{3} \rightarrow S^{2}$, and afterwards explain the small adjustments needed to handle the general case.

We begin with a continuous map $f: S^{5} \rightarrow \mathbb{C P}^{2}$ with nonzero Hopf invariant, assume that $\operatorname{Lip} f \leq 1$ and set out to prove that $\operatorname{Lip} f=1$ and that $f$ is isometric to the Hopf projection.

Step 1: Each fiber of $\boldsymbol{f}$ lies on a great 4 -sphere in $\boldsymbol{S}^{\mathbf{5}}$ In $\mathbb{C P}^{2}$, we focus on an arbitrary point $y$ and on its cut locus $Y^{\prime}=\mathbb{C P}^{1} \cong S^{2}$ at maximal constant distance $\pi / 2$ along every geodesic streaming out from $y$.

In $S^{5}$ we focus on the fiber $K=f^{-1}(y)$ and on the union of fibers $K^{\prime}=f^{-1}\left(Y^{\prime}\right)$. Let $N(K, \pi / 2)$ again denote the open $\pi / 2$ neighborhood of $K$ in $S^{5}$.

Since $\operatorname{Lip} f \leq 1$, no point in $N(K, \pi / 2)$ can map to $Y^{\prime}$.

On the other hand, some point in $S^{5}$ must map to $Y^{\prime}$, because otherwise the image of $f$ would lie in $\mathbb{C P}^{2}-Y^{\prime}$, which is an open 4-cell, and this would make $f$ homotopically trivial.

Say $f(-x) \in Y^{\prime}$.

Since $\operatorname{Lip} f \leq 1$, the point $-x$ can not lie in $N(K, \pi / 2)$, and therefore no point of $K$ can lie in the open hemisphere $N(-x, \pi / 2)$. Hence $K$ must lie in the closed hemisphere of $S^{5}$ centered at $x$.

If the point $x$ were to lie in $N(K, \pi / 2)$ then, just as in the case of maps from $S^{3} \rightarrow S^{2}$, we could construct a contractible open neighborhood $U$ of $K$ which separates it from $K^{\prime}$, and then conclude from Lemma 5 that the Hopf invariant of $f$ must be zero. Thus the point $x$ can not lie in $N(K, \pi / 2)$, and it follows that no point of $K$ can lie in the open hemisphere $N(x, \pi / 2)$. Since we already know that $K$ lies in that closed hemisphere, it follows that $K$ must lie on its boundary, which is a great 4-sphere $E S^{4}$ in $S^{5}$. 
Steps 2, 3, 4: Each fiber of $\boldsymbol{f}$ is a great circle in $\boldsymbol{S}^{\mathbf{5}}$ We then follow the argument from the case of maps from $S^{3} \rightarrow S^{2}$, using the fact that a small open neighborhood of $E S^{4}$ has trivial 1-dimensional homology, and invoke Lemma 5 once again to conclude that $K$ must in fact lie on a great 3-sphere $E S^{3} \subset E S^{4}$.

We iterate this twice more to conclude that $\mathrm{K}$ must lie on a great circle $E S^{1}$, and then copy our earlier argument from the $S^{3} \rightarrow S^{2}$ case to conclude that $K$ can not be a proper subset of that great circle, and hence must coincide with it.

Since $y$ was an arbitrary point of $\mathbb{C P}^{2}$, we now know that each fiber $K=f^{-1}(y)$ is a great circle on $S^{5}$.

Step 5: Any two great circle fibers of $\boldsymbol{f}$ are parallel to one another Consider the great circle $K=f^{-1}(y)$ and the set $K^{\prime}=f^{-1}\left(Y^{\prime}\right)$, which must be a union of great circles. Since Lip $f \leq 1$, the set $K^{\prime}$ must lie within the great 3 -sphere $S^{3}$ in $S^{5}$ which is orthogonal to $K$ and at constant maximal distance $\pi / 2$ from it.

If $K^{\prime}$ were a proper subset of $S^{3}$, we could easily construct a contractible open set $U$ in $S^{5}$ which separates $K^{\prime}$ from $K$, and then conclude from Lemma 5 that the Hopf invariant of $f$ must be zero.

Hence $K^{\prime}=S^{3}$.

We now copy the argument from the $S^{3} \rightarrow S^{2}$ case to conclude that any two great circle fibers of $f$ are parallel to one another.

Step 6: The map $\boldsymbol{f}$ is isometric to the Hopf projection Just as in the $S^{3} \rightarrow S^{2}$ case, this follows from the known fact, mentioned earlier, that any fibration of a round sphere by parallel great subspheres is isometric to the corresponding Hopf fibration.

\section{Completion of the proof of Theorem 1}

The same argument handles all the Hopf fibrations, and in each case shows that a map

$$
S^{2 n+1} \rightarrow \mathbb{C P}^{n} \quad \text { or } \quad S^{4 n+3} \rightarrow \mathbb{H P}^{n} \text { or } S^{15} \rightarrow S^{8}
$$

with nonzero Hopf invariant and Lipschitz constant less than or equal to 1 must have Lipschitz constant equal to 1 and be isometric to the corresponding Hopf projection.

This completes the proof of Theorem 1 .

Comment The set of homotopy classes of maps from $S^{2 n+1} \rightarrow \mathbb{C P}^{n}$ is in one-toone correspondence with the integers, as one sees from the homotopy sequence of the bundle $S^{1} \subset S^{2 n+1} \rightarrow \mathbb{C P}^{n}$, with the Hopf invariant providing the correspondence. 
But in the remaining cases, there are homotopically nontrivial maps which nevertheless have zero Hopf invariant.

Consider for example the Hopf fibration $S^{3} \subset S^{7} \rightarrow S^{4}$. From the homotopy sequence of this bundle and the fact that the fiber is contractible in the total space, we get

$$
\left[S^{7}, S^{4}\right] \cong \pi_{7}\left(S^{4}\right) \cong \pi_{7}\left(S^{7}\right)+\pi_{6}\left(S^{3}\right) \cong \mathbb{Z}+\mathbb{Z}_{12}
$$

The $\mathbb{Z}$-summand of $\pi_{7}\left(S^{4}\right)$ corresponds to the Hopf invariant, but the maps in the $\mathbb{Z}_{12}$-summand all have Hopf invariant zero. And likewise for all the quaternionic Hopf projections. In the one remaining case, $S^{7} \subset S^{15} \rightarrow S^{8}$, we get

$$
\left[S^{15}, S^{8}\right] \cong \pi_{15}\left(S^{8}\right) \cong \pi_{15}\left(S^{15}\right)+\pi_{14}\left(S^{7}\right) \cong \mathbb{Z}+\mathbb{Z}_{120},
$$

with the $\mathbb{Z}$-summand corresponding to the Hopf invariant, but with all the maps in the $\mathbb{Z}_{120}$-summand having Hopf invariant zero.

\section{Proof of Theorem 2}

\section{Statement of the Key Lemma}

Theorem 2 metrically characterizes the inclusion map $i: \Delta S^{n} \rightarrow S^{n} \times S^{n}$ of the diagonal as a Lipschitz constant minimizer in its homotopy class, unique up to composition with isometries of domain and range.

To prove this, we start with a map $f: \Delta S^{n} \rightarrow S^{n} \times S^{n}$ which is homotopic to $i$, assume that $\operatorname{Lip} f \leq 1$, and aim to show that $\operatorname{Lip} f=1$ and that $f$ is isometric to $i$. The basic tool is the Key Lemma, stated below.

If $x$ is any point on $S^{n}$, then $-x$ is the antipodal point, and their distance apart on $S^{n}$ is $\pi$.

Likewise, if $(x, y)$ is any point of $S^{n} \times S^{n}$, then $(-x,-y)$ will be called its antipodal point, and their distance apart on $S^{n} \times S^{n}$ is $\pi \sqrt{2}$. This is the maximum distance between any two points of $S^{n} \times S^{n}$.

Key Lemma Let $f: \Delta S^{n} \rightarrow S^{n} \times S^{n}$ be a map which is homotopic to the inclusion. Then its image $f\left(\Delta S^{n}\right)$ contains a pair of antipodal points $(x, y)$ and $(-x,-y)$ in $S^{n} \times S^{n}$.

The claim, in other words, is that the image $f\left(\Delta S^{n}\right)$ contains a pair of points at maximum distance apart in $S^{n} \times S^{n}$. In applying the Key Lemma, these two points will serve as a kind of framework, upon which the image is stretched.

The Key Lemma has a Borsuk-Ulam flavor. 


\section{Suspension}

Consider a map $\varphi: S^{m} \rightarrow S^{n}$. Then a concrete model for the suspension of $\varphi$ is the $\operatorname{map} \Sigma \varphi: S^{m+1} \rightarrow S^{n+1}$ defined by

$$
\Sigma \varphi(x \cos t, \sin t)=(\varphi(x) \cos t, \sin t),
$$

where $x \in S^{m}$ and $-\pi / 2 \leq t \leq \pi / 2$, as illustrated in the figure below.
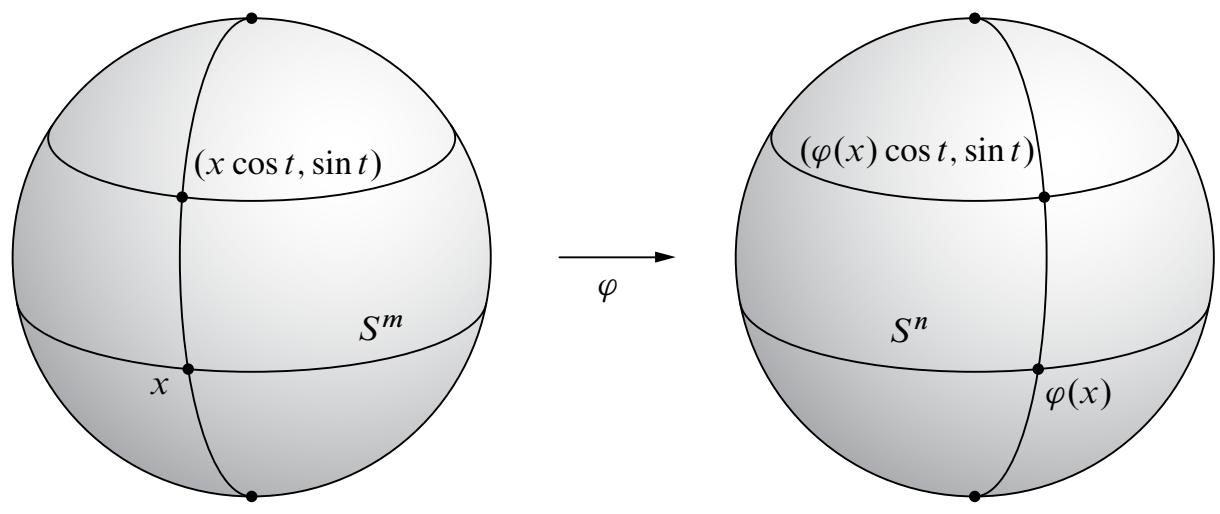

Figure 6: $\Sigma \varphi: S^{m+1} \rightarrow S^{n+1}$

The suspension of $\varphi$ takes $m$-spheres of constant latitude on $S^{m+1}$ to $n$-spheres of constant latitude on $S^{n+1}$ by rescaled copies of $\varphi$. It is almost never smooth at the north and south poles, no matter how smooth $\varphi$ is.

If $f=\left(f_{1}, f_{2}\right): S^{k} \rightarrow S^{m} \times S^{n}$, then we define the suspension of $f$ to be the map

$$
\Sigma f=\left(\Sigma f_{1}, \Sigma f_{2}\right): S^{k+1} \rightarrow S^{m+1} \times S^{n+1} .
$$

If the map $f=\left(f_{1}, f_{2}\right): \Delta S^{n} \rightarrow S^{n} \times S^{n}$ is homotopic to the inclusion, then the map $\Sigma f: \Delta S^{n+1} \rightarrow S^{n+1} \times S^{n+1}$ is also homotopic to the inclusion, with the obvious rescaling to make the suspension of $\Delta S^{n}$ into $\Delta S^{n+1}$.

\section{Plan of the proof of Theorem 2}

We start with a map $f: \Delta S^{n} \rightarrow S^{n} \times S^{n}$ which is homotopic to the inclusion, assume that $\operatorname{Lip} f \leq 1$, and aim to show that $\operatorname{Lip} f=1$ and that $f$ is isometric to the inclusion.

The argument is by induction on $n$.

We assume the truth of the Key Lemma, leave the base step $n=1$ as an exercise for the reader, and begin with the induction step as follows. 
For $n>1$, we use the Key Lemma together with the hypothesis that Lip $f \leq 1$ to desuspend $f$ to a map $f^{\prime}: \Delta S^{n-1} \rightarrow S^{n-1} \times S^{n-1}$, meaning that $\Sigma f^{\prime}=f$, such that $f^{\prime}$ is homotopic to the inclusion and satisfies $\operatorname{Lip} f^{\prime} \leq 1$.

Then by the induction hypothesis, we know that $f^{\prime}$ is isometric to the inclusion, and immediately conclude the same for $f=\Sigma f^{\prime}$.

Finally, we give the proof of the Key Lemma.

For even $n$, this is a straightforward intersection argument in $S^{n} \times S^{n}$ using homology with integer coefficients.

For odd $n$, this is an intersection argument in the symmetric product $S^{n} * S^{n}$ using homology with coefficients mod 2 .

\section{The induction step}

We assume the truth of Theorem 2 for $n-1$, and show how to prove it for $n$.

We start with a map $f: \Delta S^{n} \rightarrow S^{n} \times S^{n}$ which is homotopic to the inclusion and satisfies $\operatorname{Lip} f \leq 1$.

By the Key Lemma, the image $f\left(\Delta S^{n}\right)$ contains a pair of antipodal points $(x, y)$ and $(-x,-y)$ in $S^{n} \times S^{n}$.

Their distance apart in $S^{n} \times S^{n}$ is $\pi \sqrt{2}$, and since Lip $f \leq 1$, they must be the images of a pair of antipodal points, say $u$ and $-u$, in $\Delta S^{n}$.
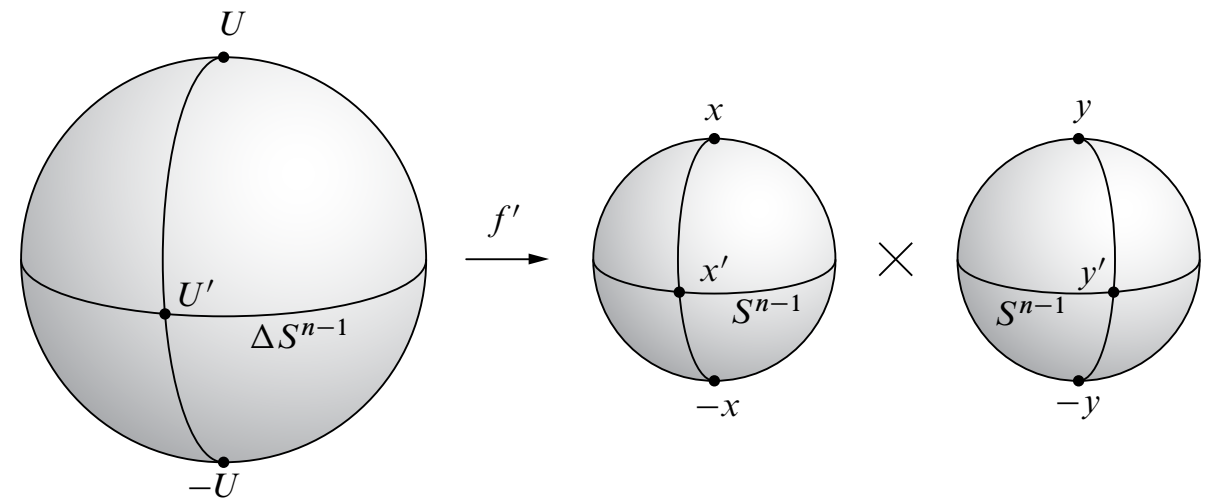

Figure 7: $f: \Delta S^{n} \rightarrow S^{n} \times S^{n}$

On $\Delta S^{n}$, each semicircle from $u$ to $-u$ is a geodesic of length $\pi \sqrt{2}$, and since Lip $f \leq 1$, it must be taken by $f$ to a geodesic, also of length $\pi \sqrt{2}$, from $(x, y)$ to $(-x,-y)$ on $S^{n} \times S^{n}$. 
The first coordinate of this image geodesic on $S^{n} \times S^{n}$ runs from $x$ to $-x$ along a semicircle on the first $S^{n}$ factor, and likewise the second coordinate runs from $y$ to $-y$ along a semicircle on the second $S^{n}$ factor.

Since Lip $f \leq 1$, the map $f$ from the semicircle on $\Delta S^{n}$ to the product of the two semicircles on $S^{n} \times S^{n}$ must be distance-preserving, with no leeway for slowing down or speeding up.

Let $\Delta S^{n-1}$ denote the equatorial $(n-1)$-sphere on $\Delta S^{n}$ with poles at $u$ and $-u$, and likewise let $S^{n-1}$ denote (ambiguously) the equatorial $(n-1)$-spheres on the two $S^{n}$ factors, with poles at $x$ and $-x$, and at $y$ and $-y$, respectively.

Let $u^{\prime}, x^{\prime}$ and $y^{\prime}$ denote the points where the three semicircles meet their respective equators, as shown in the figure above. Then $f\left(u^{\prime}\right)=\left(x^{\prime}, y^{\prime}\right)$. So we define

$$
f^{\prime}: \Delta S^{n-1} \rightarrow S^{n-1} \times S^{n-1}
$$

to be the restriction of $f$ to the equator $\Delta S^{n-1}$ on $\Delta S^{n}$.

Then we see from the above construction that $f$ is the suspension of $f^{\prime}$, that is, $f=\Sigma f^{\prime}$.

Since $\Delta S^{n-1}$ is totally geodesic in $\Delta S^{n}$, and $S^{n-1} \times S^{n-1}$ is totally geodesic in $S^{n} \times S^{n}$, the hypothesis that $\operatorname{Lip} f \leq 1$ implies that $\operatorname{Lip} f^{\prime} \leq 1$.

Furthermore, the hypothesis that $f: \Delta S^{n} \rightarrow S^{n} \times S^{n}$ is homotopic to the inclusion implies that $f^{\prime}: \Delta S^{n-1} \rightarrow S^{n-1} \times S^{n-1}$ is also homotopic to the inclusion.

The induction hypothesis, that Theorem 2 is true in dimension $n-1$, now tells us that $f^{\prime}$ must be isometric to the inclusion $i^{\prime}: \Delta S^{n-1} \rightarrow S^{n-1} \times S^{n-1}$, and it follows immediately that $f=\Sigma f^{\prime}$ must be isometric to the inclusion $i: \Delta S^{n} \rightarrow S^{n} \times S^{n}$.

This completes the proof of Theorem 2, modulo the Key Lemma.

\section{Proof of the Key Lemma for even $n$}

We start with a map $f: \Delta S^{n} \rightarrow S^{n} \times S^{n}$ which is homotopic to the inclusion, and must find a pair of antipodal points $(x, y)$ and $(-x,-y)$ in its image.

Let $a$ and $b$ denote the generators of $H_{n}\left(S^{n} \times S^{n} ; \mathbb{Z}\right)$ represented by $S^{n} \times$ point and by point $\times S^{n}$. Then $f\left(\Delta S^{n}\right)$ can be regarded as a singular $n$-cycle representing the class $a+b$.

Since $n$ is even, the intersection form on $H_{n}\left(S^{n} \times S^{n} ; \mathbb{Z}\right)$ is given by

$$
a \cdot a=b \cdot b=0, \quad a \cdot b=b \cdot a=1 .
$$




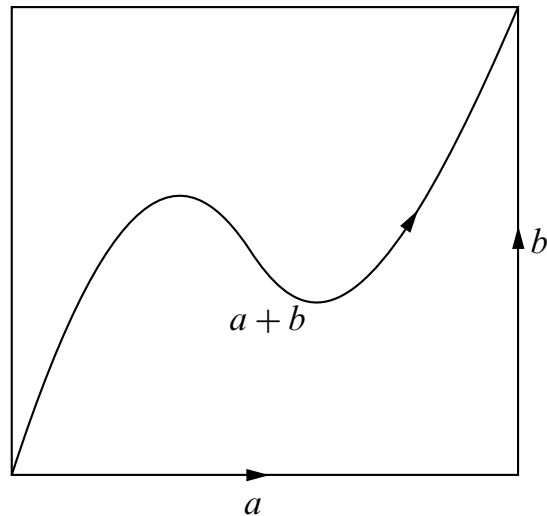

Figure 8: The $n$-cycle $f\left(\Delta S^{n}\right)$ represents the homology class $a+b$.

If $f=\left(f_{1}, f_{2}\right)$, let $-f=\left(-f_{1},-f_{2}\right)$, so that

$$
-f\left(\Delta S^{n}\right)=\left\{(-x,-y) \mid(x, y) \in f\left(\Delta S^{n}\right)\right\} .
$$

In other words, $-f\left(\Delta S^{n}\right)$ consists of the antipodes of all the points in $f\left(\Delta S^{n}\right)$.

Since $n$ is even, the oriented singular $n$-cycle $-f\left(\Delta S^{n}\right)$ represents the homology class $-a-b$.

The intersection number of the singular $n$-cycles $f\left(\Delta S^{n}\right)$ and $-f\left(\Delta S^{n}\right)$ is

$$
(a+b) \cdot(-a-b)=-2 .
$$

Hence $f\left(\Delta S^{n}\right)$ and $-f\left(\Delta S^{n}\right)$ certainly have a nonempty intersection.

Therefore, for some point $(x, y)$ in $f\left(\Delta S^{n}\right)$, the point $(-x,-y)$ is also in $f\left(\Delta S^{n}\right)$, which is precisely the claim of the Key Lemma.

Comment When $n$ is odd, the intersection form on $H_{n}\left(S^{n} \times S^{n} ; \mathbb{Z}\right)$ is given by

$$
a \cdot a=b \cdot b=0, \quad a \cdot b=1, \quad b \cdot a=-1,
$$

and the singular $n$-cycles $f\left(\Delta S^{n}\right)$ and $-f\left(\Delta S^{n}\right)$ both represent the same class $a+b$. The intersection number of these two $n$-cycles is therefore

$$
(a+b) \cdot(a+b)=0,
$$

and the preceding argument falls apart. 


\section{Rephrasing the Key Lemma}

First we restate it.

Key Lemma Let $f: \Delta S^{n} \rightarrow S^{n} \times S^{n}$ be a map which is homotopic to the inclusion. Then its image $f\left(\Delta S^{n}\right)$ contains a pair of antipodal points $(x, y)$ and $(-x,-y)$ in $S^{n} \times S^{n}$.

Then we rephrase it.

Key Lemma (rephrased) Let $f_{1}$ and $f_{2}: S^{n} \rightarrow S^{n}$ be two maps which are both homotopic to the identity. Then there are points $u$ and $v$ in $S^{n}$ such that $f_{1}(u)$ and $f_{1}(v)$ are antipodal, and at the same time $f_{2}(u)$ and $f_{2}(v)$ are also antipodal.

To match the two versions, put $f(x, x)=\left(f_{1}(x), f_{2}(x)\right)$.

In the two hypotheses, $f$ is homotopic to the inclusion if and only if $f_{1}$ and $f_{2}$ are both homotopic to the identity. And in the two conclusions, $f\left(\Delta S^{n}\right)$ contains a pair of antipodal points, call them $f(u, u)=\left(f_{1}(u), f_{2}(u)\right)$ and $f(v, v)=\left(f_{1}(v), f_{2}(v)\right)$, if and only if $f_{1}(u)$ and $f_{1}(v)$ are antipodal, and at the same time $f_{2}(u)$ and $f_{2}(v)$ are antipodal.

The proof of the Key Lemma which we give here is due to Dennis Sullivan. It begins with the alternative phrasing above, and then moves the scene of action from the cartesian product $S^{n} \times S^{n}$ down to the symmetric product $S^{n} * S^{n}$, in which every point $(u, v)$ is identified with its "flip" $(v, u)$.

The virtue of this move is that the image of the "antidiagonal" in $S^{n} * S^{n}$ will be seen to have self-intersection number $1(\bmod 2)$ there, independent of the parity of $n$.

To prepare for the argument, we pause to discuss the geometries of both the cartesian and symmetric products.

\section{Geometry of the cartesian product $S^{n} \times S^{n}$}

Let

$$
\begin{aligned}
& D=\Delta S^{n}=\text { diagonal } n \text {-sphere }=\left\{(x, x) \mid x \in S^{n}\right\} \subset S^{n} \times S^{n}, \\
& A=\text { antidiagonal } n \text {-sphere }=\left\{(x,-x) \mid x \in S^{n}\right\} \subset S^{n} \times S^{n} .
\end{aligned}
$$

Each of $D$ and $A$ is the focal locus of the other in $S^{n} \times S^{n}$, and the isometry $\varphi: S^{n} \times S^{n} \rightarrow S^{n} \times S^{n}$ defined by $\varphi(x, y)=(x,-y)$ interchanges them. 
The diagonal $D$ and antidiagonal $A$ are homologous to one another when $n$ is odd, but not when $n$ is even.

The set $U=\left\{(x, y) \in S^{n} \times S^{n} \mid x \cdot y=0\right\}$ is a copy of the Stiefel manifold $V_{2} \mathbb{R}^{n+1}$ of orthonormal two-frames in $R^{n+1}$, and is situated halfway between $D$ and $A$ in $S^{n} \times S^{n}$.

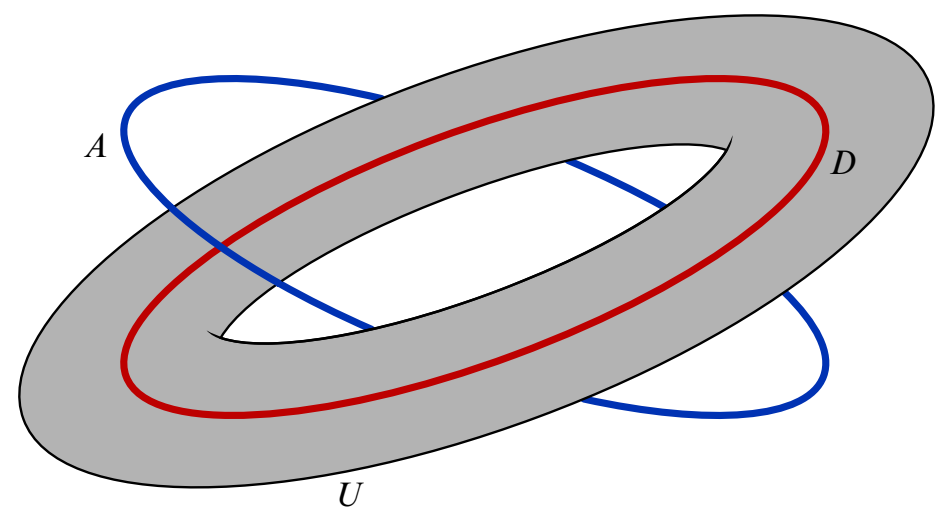

Figure 9: The hypersurface $U$ is halfway between $D$ and $A$ in $S^{n} \times S^{n}$

The inner product function IP: $S^{n} \times S^{n} \rightarrow \mathbb{R}$ defined by $\operatorname{IP}(x, y)=x \cdot y$ takes values in the interval $[-1,1]$, and we have

$$
D=\mathrm{IP}^{-1}(1), \quad U=\mathrm{IP}^{-1}(0), \quad A=\mathrm{IP}^{-1}(-1) .
$$

The level sets $\operatorname{IP}^{-1}(t)$ for $-1<t<1$ are all homeomorphic to one another, and together foliate the complement of $D$ and $A$ in $S^{n} \times S^{n}$, collapsing to $D$ at one end and to $A$ at the other.

The sets $N(D)=\mathrm{IP}^{-1}([0,1])$ and $N(A)=\mathrm{IP}^{-1}([-1,0])$ are closed tubular neighborhoods of $D$ and $A$ in $S^{n} \times S^{n}$ which share $U$ as a common boundary. They are both copies of the unit disk bundle of the tangent bundle of $S^{n}$.

\section{The symmetric product $S^{n} * S^{n}$}

Consider the involution $\sigma: S^{n} \times S^{n} \rightarrow S^{n} \times S^{n}$ defined by $\sigma(x, y)=(y, x)$.

The symmetric product $S^{n} * S^{n}$ is obtained from $S^{n} \times S^{n}$ by dividing out by this involution,

$$
S^{n} * S^{n}=\frac{S^{n} \times S^{n}}{(x, y) \sim(y, x)}
$$

Let $p: S^{n} \times S^{n} \rightarrow S^{n} * S^{n}$ be the projection map, and write $p(x, y)=[x, y]$. 
Each level set of the inner product function IP: $S^{n} \times S^{n} \rightarrow[-1,1]$ is invariant under the involution $\sigma$, and hence the decomposition of $S^{n} \times S^{n}$ into these level sets projects under $p$ to a corresponding decomposition of $S^{n} * S^{n}$.

The fixed point set of $\sigma$ is the diagonal $n$-sphere $D$, which projects one-to-one to its image $p(D)=D^{\prime}$ in $S^{n} * S^{n}$. At the other extreme is the antidiagonal $A$ in $S^{n} \times S^{n}$, which projects two-to-one to its image $p(A)=A^{\prime}$, a copy of $\mathbb{R} \mathbb{P}^{n}$, in $S^{n} * S^{n}$.

The tubular neighborhoods $N(D)$ and $N(A)$ in $S^{n} \times S^{n}$ project to tubular neighborhoods $N\left(D^{\prime}\right)$ and $N\left(A^{\prime}\right)$ in $S^{n} * S^{n}$.

The symmetric product $S^{1} * S^{1}$ is a Möbius band, with $D^{\prime}$ as its boundary, while the symmetric product $S^{2} * S^{2}$ is homeomorphic to $\mathbb{C P}^{2}$.

By contrast, the symmetric product $S^{n} * S^{n}$ fails to be a manifold along $D^{\prime}$ starting with $n=3$. Nevertheless, for all $n,\left(S^{n} * S^{n}\right)-D^{\prime}$ is a (noncompact) manifold containing $A^{\prime}$ as a submanifold.

Since the involution $\sigma$ of $S^{n} \times S^{n}$ is orientation-preserving for even $n$ and orientationreversing for odd $n$, the symmetric product $S^{n} * S^{n}$ is orientable for even $n$ and nonorientable for odd $n$.

By contrast, the image $A^{\prime}$ of the antidiagonal is homeomorphic to $\mathbb{R} \mathbb{P}^{n}$, and is therefore nonorientable for even $n$ and orientable for odd $n$.

Thus the symmetric product $S^{n} * S^{n}$ has some prominent nonorientable feature for all $n$.

\section{Self-intersection number of the antidiagonal in $S^{n} * S^{n}$}

Recall that in $S^{n} \times S^{n}$,

- when $n$ is even, the diagonal $D$ has self-intersection number 2 and the antidiagonal $A$ has self-intersection number -2 ;

- when $n$ is odd, $D$ and $A$ each have self-intersection number 0 .

Lemma 6 Regardless of the parity of $n$, the antidiagonal $A^{\prime}=p(A)$ in $\left(S^{n} * S^{n}\right)-D^{\prime}$ has self-intersection number $1(\bmod 2)$.

When $n=1$ this is easy to see visually, since $S^{1} * S^{1}$ is a Möbius band, while $A^{\prime}$ is the circle running along the middle of the band.

To prove the lemma in general, we will describe a concrete perturbation of $A^{\prime}$ in $\left(S^{n} * S^{n}\right)-D^{\prime}$ which meets $A^{\prime}$ transversally in just one point. 
To that end, let $f: S^{n} \rightarrow S^{n}$ be a diffeomorphism with fixed points at the north and south poles, but otherwise moving each point of $S^{n}$ slightly southwards along its circle of longitude. We want to choose $f$ to satisfy the following two conditions:

(1) the differential of $f$ at the north pole is expansive, and at the south pole contractive;

(2) the behavior of $f$ is related to the antipodal map as in the following diagram, which shows a typical great circle of longitude.

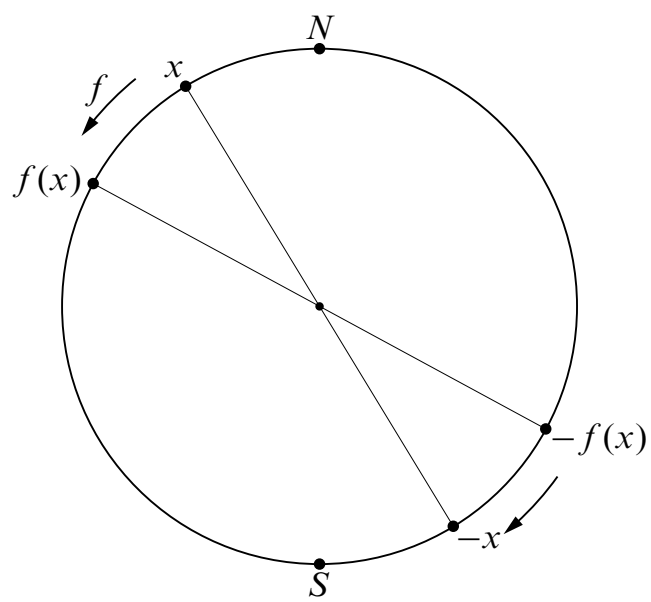

Figure 10: Required behavior of $f$

We are requiring that $f(-f(x))=-x$, or in other words, that the map $-f$ be an involution.

We intend that $f: S^{n} \rightarrow S^{n}$ should be the same on every great semicircle of longitude, and construct such a map as follows.

First we redraw the above circle of longitude on $S^{n}$, focus on its "left half", and parametrize this from 0 in the north to 1 in the south.

We see in Figure 11 that the point $-f(x)$ corresponds to the point $1-f(x)$ in this parametrization, and likewise the point $-x$ corresponds to $1-x$.

So, focusing on the left semicircle, and thinking of $f$ as a map from $[0,1]$ to itself, we are requiring in condition (2) above that

(2') $f(1-f(x))=1-x$. 


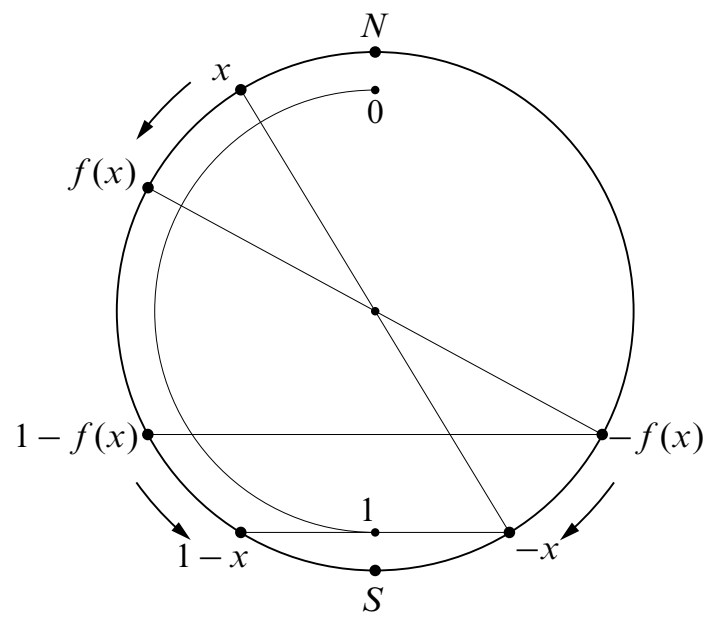

Figure 11: Behavior of reparametrized $f$

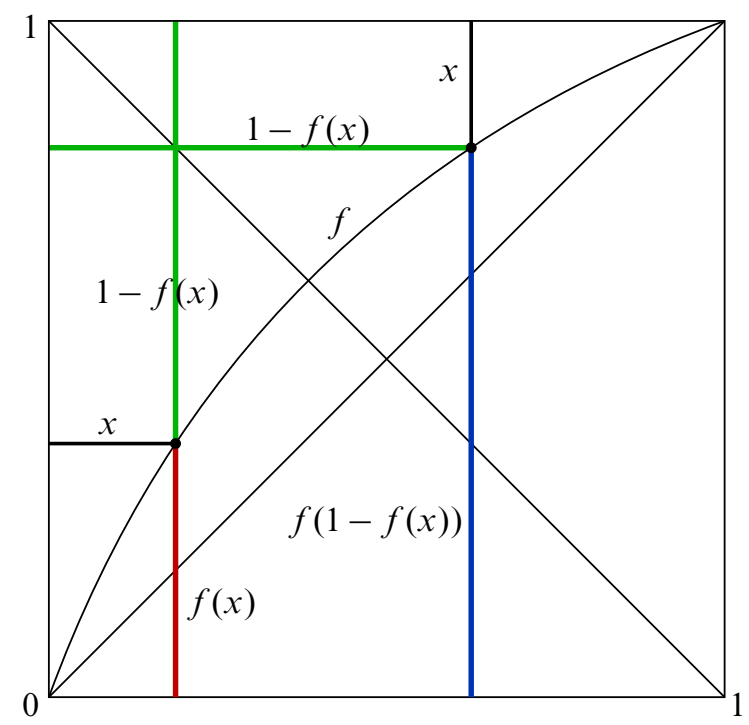

Figure 12: Guide for constructing $f$

To construct such a function $f$, we are guided by Figure 12, in which we show the graph of $f$ inside the square $[0,1] \times[0,1]$.

We insist that the graph of $f$ should be invariant under reflection about the diagonal of slope -1 , and will show that this guarantees condition (2'). 
To that end, start with the point $(x, f(x))$ on the graph. In the diagram, we show $x$ as a horizontal segment in black, $f(x)$ as a vertical segment in red, and then $1-f(x)$ as a vertical segment in green above it.

Now reflect in the diagonal of slope -1 . Then $1-f(x)$ reappears as a horizontal segment in green, and due to the symmetry, its right hand end point is still on the graph of $f$.

Hence $f(1-f(x))$ appears as a vertical segment in blue.

Then $1-f(1-f(x))$ appears directly above it as a vertical segment in black which, thanks again to the reflective symmetry, has length $x$. That is, $1-f(1-f(x))=x$, which is just a transposed version of condition (2').

To take care of condition (1), we simply make $f^{\prime}(0)>1$. Then by the reflective symmetry we have $f^{\prime}(1)=1 / f^{\prime}(0)<1$. For example, in Figure 12 , we have $f^{\prime}(0)=2$ and $f^{\prime}(1)=1 / 2$.

We now define $f: S^{n} \rightarrow S^{n}$ by copying the map $f$ on every great semicircle of longitude from the north pole $N$ to the south pole $S$. We guarantee the differentiability of this $f$ at $N$ and $S$ by making the map $f:[0,1] \rightarrow[0,1]$ linear near 0 , and hence by reflection, also near 1, as shown in Figure 12.

With such a map $f: S^{n} \rightarrow S^{n}$ in hand, we complete the proof of Lemma 6 as follows. The antidiagonal $n$-sphere $A=\{(x,-x)\}$ in $S^{n} \times S^{n}$ projects down by $p$ to the antidiagonal real projective $n$-space $A^{\prime}=\{[x,-x]\}$ in $S^{n} * S^{n}$.

Up in $S^{n} \times S^{n}$, consider the smooth $n$-sphere $A_{f}=\{(x,-f(x)\}$. If $f$ is close to the identity, then $A_{f}$ is a slight perturbation of $A$, which by condition (1) above meets $A$ transversally in the two points $(N, S)$ and $(S, N)$.

Furthermore, by condition (2), for each point $(x,-f(x))$ of $A_{f}$, the point $(-f(x), x)$ is also in $A_{f}$, and so $A_{f}$ is invariant under the involution $\sigma$.

Therefore the map $p: A_{f} \rightarrow p\left(A_{f}\right)=A_{f}^{\prime}$ is a double covering, whose image is a slight perturbation of $A^{\prime}$, and which meets it transversally in the single point $[N, S]=[S, N]$. By construction of $f$, this perturbation of $A^{\prime}$ takes place entirely in $\left(S^{n} * S^{n}\right)-D^{\prime}$.

This completes the proof of Lemma 6.

\section{Proof of the Key Lemma}

In order to prove the rephrased version of the Key Lemma, we start with the two maps $f_{1}$ and $f_{2}: S^{n} \rightarrow S^{n}$, both homotopic to the identity, and must find points $u$ and $v$ 
in $S^{n}$ such that $f_{1}(u)$ and $f_{1}(v)$ are antipodal, and at the same time $f_{2}(u)$ and $f_{2}(v)$ are also antipodal.

To that end, define $F_{1}$ and $F_{2}: S^{n} * S^{n} \rightarrow S^{n} * S^{n}$ by

$$
F_{1}([u, v])=\left[f_{1}(u), f_{1}(v)\right], \quad F_{2}([u, v])=\left[f_{2}(u), f_{2}(v)\right] .
$$

We will show that

$$
F_{1}^{-1}\left(A^{\prime}\right) \cap F_{2}^{-1}\left(A^{\prime}\right) \neq \varnothing .
$$

Suppose, to the contrary, that the inverse images $F_{1}^{-1}\left(A^{\prime}\right)$ and $F_{2}^{-1}\left(A^{\prime}\right)$ are disjoint. Choose an open tubular neighborhood $N^{\prime}$ of $A^{\prime}$ in $S^{n} * S^{n}-D^{\prime}$ such that

$$
F_{1}^{-1}\left(N^{\prime}\right) \cap F_{2}^{-1}\left(N^{\prime}\right)=\varnothing .
$$

In the following argument, all homology and cohomology will be understood to have $\mathbb{Z}_{2}$ coefficients. The symbol $H_{c}^{2 n}$ denotes cohomology with compact supports.

Using the terminology and results of Eilenberg-Steenrod XI.6, the pair $\left(S^{n} * S^{n}, D^{\prime}\right)$ is a relative $2 n-$ manifold. The fact that $S^{n} * S^{n}-D^{\prime}$ is connected implies that

$$
H^{2 n}\left(S^{n} * S^{n}, D^{\prime}\right) \cong \mathbb{Z}_{2}, \quad H_{c}^{2 n}\left(S^{n} * S^{n}-D^{\prime}\right) \cong \mathbb{Z}_{2},
$$

and the inclusion $\left(S^{n} * S^{n}-D^{\prime}, \varnothing\right) \subset\left(S^{n} * S^{n}, D^{\prime}\right)$ induces an isomorphism

$$
H_{c}^{2 n}\left(S^{n} * S^{n}-D^{\prime}\right) \rightarrow H^{2 n}\left(S^{n} * S^{n}, D^{\prime}\right) .
$$

Consider the compact smooth submanifold $A^{\prime} \subset S^{n} * S^{n}-D^{\prime}$. Let $\omega$ be an $n$-dimensional cochain representing the Poincaré dual of $A^{\prime}$ in the cohomology ring $H_{c}^{2 n}\left(S^{n} * S^{n}-D^{\prime}\right)$, that is, $\omega$ represents the Thom class of the normal bundle of $A^{\prime}$. We may assume that $\omega$ is supported in the open tubular neighborhood $N^{\prime}$ of $A^{\prime}$.

We saw in the previous section that $A^{\prime}$ has nonzero self-intersection number mod 2 in $S^{n} * S^{n}-D^{\prime}$. It follows from Poincaré duality that the cup product $[\omega] \cup[\omega]$ is nonzero in $H_{c}^{2 n}\left(S^{n} * S^{n}-D^{\prime}\right)$. Using the inclusion map, we see that $[\omega] \cup[\omega]$ is also nonzero in $H^{2 n}\left(S^{n} * S^{n}, D^{\prime}\right)$.

Since $f_{1}$ and $f_{2}: S^{n} \rightarrow S^{n}$ are both homotopic to the identity, it follows that $F_{1}$ and $F_{2}: S^{n} * S^{n} \rightarrow S^{n} * S^{n}$ are also both homotopic to the identity through maps which always take the singular locus $D^{\prime}$ to itself (though not keeping it pointwise fixed). Therefore

$$
F_{1}^{*}[\omega] \cup F_{2}^{*}[\omega]=[\omega] \cup[\omega]=1
$$

in $H^{2 n}\left(S^{n} * S^{n}, D^{\prime}\right) \cong \mathbb{Z}_{2}$. 
Since the support of $\omega$ is contained in $N^{\prime}$, and we have seen $F_{1}^{-1}\left(N^{\prime}\right) \cap F_{2}^{-1}\left(N^{\prime}\right)=\varnothing$, it follows that the cochains $F_{1}^{*} \omega$ and $F_{2}^{*} \omega$ have disjoint supports. Therefore

$$
F_{1}^{*}[\omega] \cup F_{2}^{*}[\omega]=0 .
$$

This contradiction shows that

$$
F_{1}^{-1}\left(A^{\prime}\right) \cap F_{2}^{-1}\left(A^{\prime}\right) \neq \varnothing .
$$

Now let $[u, v]$ be a point in this intersection.

The fact that $F_{1}([u, v])=\left[f_{1}(u), f_{1}(v)\right]$ lies in $A^{\prime}$ tells us that $f_{1}(u)$ and $f_{1}(v)$ are antipodal.

The fact that $F_{2}([u, v])=\left[f_{2}(u), f_{2}(v)\right]$ lies in $A^{\prime}$ tells us that $f_{2}(u)$ and $f_{2}(v)$ are antipodal.

This is exactly the claim of the Key Lemma, and so completes its proof, and with it the proof of Theorem 2 .

\section{Proof of Theorem 3}

\section{Equivalence of Hopf vector fields}

We will make use of the following in the proof of Theorem 3.

An orthogonal complex structure $J$ on Euclidean space $\mathbb{R}^{2 n+2}$ is an element of the group $S O(2 n+2)$ such that $J^{2}=-$ Identity. Decomposing $\mathbb{R}^{2 n+2}$ into an orthogonal direct sum of 2-planes invariant under $J$, we note that

(i) $x \cdot J(x)=0$ for all $x \in \mathbb{R}^{2 n+2}$;

(ii) any two orthogonal complex structures on $\mathbb{R}^{2 n+2}$ are conjugate in $O(2 n+2)$.

A Hopf vector field on $S^{2 n+1}$ is the same thing as the restriction to $S^{2 n+1}$ of an orthogonal complex structure on $\mathbb{R}^{2 n+2}$.

Suppose that $J$ and $J^{\prime}$ are any two orthogonal complex structures on $\mathbb{R}^{2 n+2}$, and that $g$ is an element of $O(2 n+2)$, thanks to (ii) above, such that $g J=J^{\prime} g$. Then $(g, g)$ is an isometry of the unit tangent bundle $U S^{2 n+1}$ taking the graph $V$ of the restriction of $J$ to $S^{2 n+1}$ to the graph $V^{\prime}$ of the corresponding restriction of $J^{\prime}$, since

$$
(g, g)(x, J(x))=(g(x), g J(x))=\left(g(x), J^{\prime} g(x)\right)=\left(y, J^{\prime}(y)\right) .
$$

In other words, any two Hopf cross sections $V\left(S^{2 n+1}\right)$ and $V^{\prime}\left(S^{2 n+1}\right)$ of $U S^{2 n+1}$ can be taken to one another by an isometry of this unit tangent bundle. 


\section{Proof of Theorem 3}

Let $v$ be a Hopf vector field on $S^{2 n+1}$, obtained as the restriction of the orthogonal complex structure $J$ on $R^{2 n+2}$. Let $V$ be the corresponding cross-section of $U S^{2 n+1}$, and denote by $i: V\left(S^{2 n+1}\right) \rightarrow U S^{2 n+1}$ the inclusion map. The claim of Theorem 3 is that if the map $f: V\left(S^{2 n+1}\right) \rightarrow U S^{2 n+1}$ is homotopic to the inclusion and has Lipschitz constant less than or equal to 1 , then its Lipschitz constant equals 1 and $f$ is isometric to the inclusion.

The composite inclusion $V\left(S^{2 n+1}\right) \subset U S^{2 n+1} \subset S^{2 n+1} \times S^{2 n+1}$ is isometric to the inclusion of the diagonal $\Delta S^{2 n+1} \subset S^{2 n+1} \times S^{2 n+1}$, since the restriction of $J$ to $S^{2 n+1}$ is an isometry.

The composite map $f: V\left(S^{2 n+1}\right) \rightarrow U S^{2 n+1} \subset S^{2 n+1} \times S^{2 n+1}$ is homotopic to the inclusion of $V\left(S^{2 n+1}\right)$ into $S^{2 n+1} \times S^{2 n+1}$, and still has Lipschitz constant less than or equal to 1 there. So by Theorem 2, the composite map $f$ must have Lipschitz constant equal to 1 and be isometric to the inclusion of $V\left(S^{2 n+1}\right)$ into $S^{2 n+1} \times S^{2 n+1}$.

Thus the image under $f$ of $V\left(S^{2 n+1}\right)$ in $S^{2 n+1} \times S^{2 n+1}$ must be the graph $V^{\prime}$ of an orientation-preserving isometry $J^{\prime}: S^{2 n+1} \rightarrow S^{2 n+1}$ such that $x \cdot J^{\prime}(x)=0$ for all $x$ in $S^{2 n+1}$. In other words, $f$ takes the Hopf cross-section $V\left(S^{2 n+1}\right)$ to another Hopf cross-section $V^{\prime}\left(S^{2 n+1}\right)$ in $U S^{2 n+1}$.

Since both $V\left(S^{2 n+1}\right)$ and $V^{\prime}\left(S^{2 n+1}\right)$ are round $2 n+1$-spheres of radius $\sqrt{2}$, and $f$ has Lipschitz constant less than or equal to 1 , the map $f: V\left(S^{2 n+1}\right) \rightarrow V^{\prime}\left(S^{2 n+1}\right)$ must be an isometry.

We saw in the previous section that there is an isometry of $U S^{2 n+1}$ to itself which takes $V\left(S^{2 n+1}\right)$ to $V^{\prime}\left(S^{2 n+1}\right)$. It follows that $f: V\left(S^{2 n+1}\right) \rightarrow U S^{2 n+1}$ is isometric to the inclusion $i: V\left(S^{2 n+1}\right) \rightarrow U S^{2 n+1}$, completing the proof of Theorem 3 .

So we see that Theorem 3 is a direct consequence of Theorem 2.

\section{Comment on Theorem 3}

There are really two distinct Riemannian metrics on the unit tangent bundle of a sphere.

The first, which we have been using, views

$$
U S^{n}=\left\{(x, y) \mid x, y \in S^{n}, x \cdot y=0\right\} \subset S^{n} \times S^{n},
$$

takes the usual product metric on $S^{n} \times S^{n}$, and then gives to $U S^{n}$ the Riemannian metric induced by this inclusion. 
The second, due to Sasaki [28], applies to the tangent bundle $T M$ of any Riemannian manifold $M$. If $(x(t), v(t))$ is a curve in $T M$, then the length of the tangent vector to this curve is taken to be

$$
\left(\left|x^{\prime}(t)\right|^{2}+\left|v^{\prime}(t)\right|^{2}\right)^{1 / 2}
$$

where $x^{\prime}(t)$ is the tangent vector to the curve $x(t)$ in $M$, where $v^{\prime}(t)$ is the covariant derivative of the vector field $v(t)$ along the curve $x(t)$, and the norms of these vectors are measured in the given Riemannian metric on $M$.

As a result, if $v(t)$ is a parallel vector field along the curve $x(t)$ in $M$, meaning that the covariant derivative $v^{\prime}(t)=0$, then the length of the tangent vector to the curve $(x(t), v(t))$ in $T M$ is simply the length $\left|x^{\prime}(t)\right|$ of the tangent vector to the curve $x(t)$ in $M$.

For example, if $M$ is the unit circle $S^{1}$ in $\mathbb{R}^{2}$, and if

$$
x(t)=(\cos t, \sin t), \quad v(t)=(-\sin t, \cos t),
$$

then not only is $x(t)$ a unit speed curve in $M$, but also $(x(t), v(t))$ is a unit speed curve in $T M$, since $v(t)$ is parallel along $x(t)$. In other words, the fact that $v(t)$ is, to the naked eye, spinning around just as fast as $x(t)$, is forgiven, and the length of the loop $(x(t), v(t))$ in $T M$ is just $2 \pi$, as opposed to $2 \pi \sqrt{2}$.

The same thing happens with the Sasaki metric on the unit tangent bundle $U S^{n}$ of $S^{n}$. If $x(t)$ travels at unit speed once around a great circle in $S^{n}$, and if $v(t)=x^{\prime}(t)$ is its velocity vector, then the curve $(x(t), v(t))$ in $U S^{n}$ also travels at unit speed in $U S^{n}$, and so has length $2 \pi$. By contrast, in the "product metric" on $U S^{n}$ inherited from its natural inclusion in $S^{n} \times S^{n}$, this loop has length $2 \pi \sqrt{2}$.

This is the only difference between the two competing metrics on $U S^{n}$ : you pass from the product metric to the Sasaki metric by reducing lengths by a factor of $\sqrt{2}$ in the direction of the above "geodesic flow", while preserving lengths in the orthogonal direction.

It would be sensible to check the validity of Theorem 3 using the Sasaki metric on the unit tangent bundle $U S^{2 n+1}$, but we have not done this yet.

\section{Proof of Theorem 4}

\section{Setup}

The Stiefel manifold $V_{2} \mathbb{R}^{4}$ is the set of orthonormal 2-frames in 4-space,

$$
V_{2} \mathbb{R}^{4}=\left\{(x, y) \mid x \in S^{3}, y \in S^{3}, x \cdot y=0\right\},
$$


and we give it the Riemannian metric inherited from its inclusion in $S^{3} \times S^{3}$.

The Grassmann manifold $G_{2} \mathbb{R}^{4}$ is the set of oriented 2-planes through the origin in 4-space. We identify it with the set of unit decomposable 2-vectors in the exterior product $\Lambda^{2} \mathbb{R}^{4}$, a 6 -dimensional Euclidean space, and give it the resulting Riemannian metric.

Fact (Gluck and Warner [13]) A 2-vector in 4-space is decomposable if and only if it has equal length projections into the +1 and -1 eigenspaces $E_{+}^{3}$ and $E_{-}^{3}$ of the Hodge star operator on $\Lambda^{2} \mathbb{R}^{4}$.

From this fact, it follows that

$$
G_{2} \mathbb{R}^{4}=S_{+}^{2}(1 / \sqrt{2}) \times S_{-}^{2}(1 / \sqrt{2}) \subset E_{+}^{3} \oplus E_{-}^{3}=\Lambda^{2} \mathbb{R}^{4},
$$

the product of the $2-$ spheres of radius $1 / \sqrt{2}$ in $E_{+}^{3}$ and $E_{-}^{3}$.

The projection map $p: V_{2} \mathbb{R}^{4} \rightarrow G_{2} \mathbb{R}^{4}$ takes $(x, y) \rightarrow(x \wedge y) /\|x \wedge y\|$. It is a Riemannian submersion, and thus has Lipschitz constant 1.

Theorem 4 asserts that any map homotopic to the Stiefel projection has Lipschitz constant greater than or equal to 1 , with equality if and only if the map is isometric to this projection.

In other words, the Stiefel projection is, up to isometries of domain and range, the unique Lipschitz constant minimizer in its homotopy class.

To prove this theorem, we will observe within the Stiefel projection $V_{2} \mathbb{R}^{4} \rightarrow G_{2} \mathbb{R}^{4}$ two families of Hopf projections $S^{3} \rightarrow S^{2}$, whose Lipschitz minimality, unique up to isometries of domain and range, was established in Theorem 1. They provide the framework for the proof.

\section{An alternative view of the Stiefel projection}

The Stiefel manifold $V_{2} \mathbb{R}^{4}$ is the same as the unit tangent bundle $U S^{3}$ of the 3 -sphere. This bundle is trivial topologically (though not metrically), and has two common sense trivializations, $U S^{3} \rightarrow S^{3} \times S^{2}$, given by

$$
(x, y) \rightarrow\left(x, y x^{-1}\right), \quad(x, y) \rightarrow\left(x, x^{-1} y\right),
$$

using multiplication of unit quaternions, and thinking of $S^{2}$ as the space of purely imaginary unit quaternions.

Packaging these two trivializations together yields a map

$$
V_{2} \mathbb{R}^{4}=U S^{3} \rightarrow S^{2} \times S^{2}, \quad(x, y) \rightarrow\left(y x^{-1}, x^{-1} y\right),
$$


which is a copy of the Stiefel projection $p: V_{2} \mathbb{R}^{4} \rightarrow G_{2} \mathbb{R}^{4}$, scaled up by the linear factor $\sqrt{2}$.

This version of the Stiefel projection $p$ has Lipschitz constant $\sqrt{2}$, and we will use it in what follows.

\section{Copies of the complex Grassmannian $G_{1} \mathbb{C}^{2}$ inside $G_{2} \mathbb{R}^{4}$}

It is easy to see that on $\mathbb{R}^{4}$, all orthogonal complex structures are given by left or right multiplication by a purely imaginary unit quaternion. To be specific, let us use left multiplication by the purely imaginary unit quaternion $u$ to regard $\mathbb{R}^{4}$ as $\mathbb{C}^{2}$.

The corresponding complex Grassmannian $G_{1} \mathbb{C}^{2}$ consists of all complex lines in $\mathbb{C}^{2}$ through the origin. To real eyes, each such complex line is a 2 -plane through the origin, with a natural orientation given by the ordered basis $x, u x$ for any unit vector $x$ therein.

Using the alternative version $p(x, y)=\left(y x^{-1}, x^{-1} y\right)$ of the Stiefel projection, we have $p(x, u x)=\left(u, x^{-1} u x\right)$, which tells us that

$$
G_{1} \mathbb{C}^{2}=u \times S^{2} \subset S^{2} \times S^{2}=G_{2} \mathbb{R}^{4},
$$

a "vertical" 2-sphere in $S^{2} \times S^{2}$. The inverse image $p^{-1}\left(G_{1} \mathbb{C}^{2}\right)$ up in $V_{2} \mathbb{R}^{4}$ is the subset

$$
{ }_{u} V=\left\{(x, u x) \mid x \in S^{3}\right\} .
$$

It is a round, totally geodesic 3 -sphere of radius $\sqrt{2}$, and is the graph of the corresponding Hopf vector field.

The restriction $p:{ }_{u} V \rightarrow G_{1} \mathbb{C}^{2}$ of the Stiefel projection is just a copy of the Hopf projection, scaled up by a factor $\sqrt{2}$.

Varying the choice of purely imaginary unit quaternion $u$ gives us all possible "vertical" 2-spheres $u \times S^{2}$ as the corresponding $G_{1} \mathbb{C}^{2}$ inside our $S^{2} \times S^{2}$ picture of $G_{2} \mathbb{R}^{4}$. Each one serves as the base space of a Hopf projection, as above.

Similarly, fixing an orthogonal complex structure on $\mathbb{R}^{4}$ via right multiplication by the purely imaginary unit quaternion $v$, we get the corresponding

$$
G_{1} \mathbb{C}^{2}=S^{2} \times v \subset S^{2} \times S^{2}=G_{2} \mathbb{R}^{4},
$$

a "horizontal" 2-sphere in $S^{2} \times S^{2}$.

The inverse image $p^{-1}\left(G_{1} \mathbb{C}^{2}\right)$ up in $V_{2} \mathbb{R}^{4}$ is the subset

$$
V_{v}=\left\{(x, x v) \mid x \in S^{3}\right\},
$$


and again, the restriction $p: V_{v} \rightarrow G_{1} \mathbb{C}^{2}$ of the Stiefel projection is a scaled-up copy of the Hopf projection.

Varying the choice of $v$ gives us all possible "horizontal" 2 -spheres $S^{2} \times v$ as the corresponding $G_{1} \mathbb{C}^{2}$ inside our $S^{2} \times S^{2}$ picture of $G_{2} \mathbb{R}^{4}$, again with each serving as the base space of a Hopf projection.

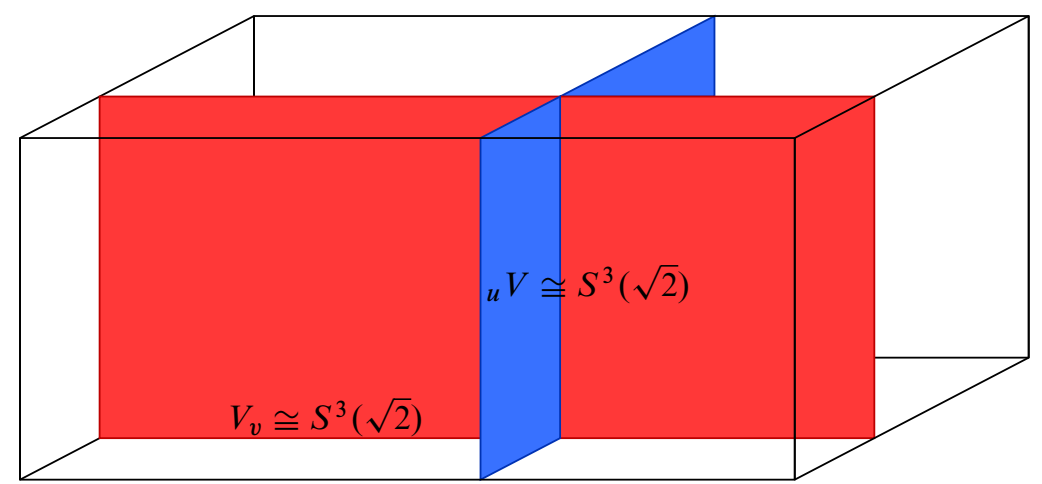

$V_{2} R^{4}$

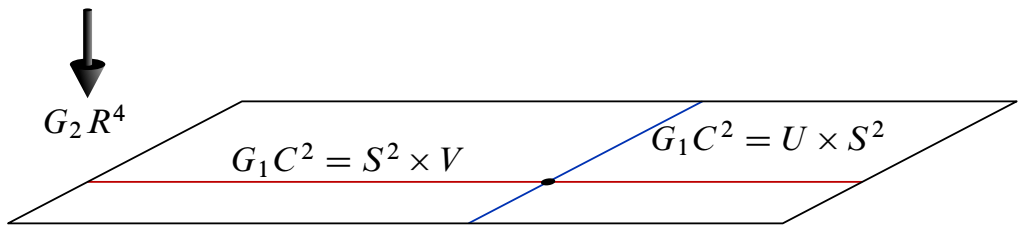

Figure 13: Two copies of $G_{1} \mathbb{C}^{2}$ inside $G_{2} \mathbb{R}^{4}$, and their inverse images in $V_{2} \mathbb{R}^{4}$

\section{Proof of Theorem 4}

We start with the Stiefel projection $p: V_{2} \mathbb{R}^{4} \rightarrow G_{2} \mathbb{R}^{4}=S^{2} \times S^{2}$, which is a scaled-up Riemannian submersion with Lipschitz constant $\sqrt{2}$.

Then we consider another map $f: V_{2} \mathbb{R}^{4} \rightarrow G_{2} \mathbb{R}^{4}$ which is homotopic to $p$ with Lipschitz constant less than or equal to $\sqrt{2}$, and must show that $f$ has Lipschitz constant equal to $\sqrt{2}$, and agrees with $p$ up to composition with isometries of domain and range.

The isometries of the domain $V_{2} \mathbb{R}^{4}$ are known to preserve the Stiefel fibers (Gluck and Ziller [16]), so somewhere in the argument we will have to show that these are also the fibers of $f$, and we take this as a hint.

Fix a purely imaginary unit quaternion $v$, and use right multiplication by $v$ to impose an orthogonal complex structure on $\mathbb{R}^{4}$, so that we can regard it as $\mathbb{C}^{2}$. 
Then consider again the restriction $p: V_{v} \rightarrow G_{1} \mathbb{C}^{2} \subset G_{2} \mathbb{R}^{4}$ of the Stiefel projection. Let $p_{1}$ and $p_{2}$ denote the compositions of $p$ with the projections of $G_{2} \mathbb{R}^{4}=S^{2} \times S^{2}$ to its first and second factors.

Likewise, let $f_{1}$ and $f_{2}$ denote the corresponding compositions of $f$ with these two projections.

Now compare the restrictions

$$
p_{1}, f_{1}: V_{v} \cong S^{3}(\sqrt{2}) \rightarrow S^{2} .
$$

Since $p$ and $f$ are homotopic, so are the above restrictions of $p_{1}$ and $f_{1}$. The restricted $p_{1}$ is a Hopf projection, scaled up by $\sqrt{2}$, with Lipschitz constant $\sqrt{2}$, while the restricted $f_{1}$ has Lipschitz constant less than or equal to $\sqrt{2}$.

By Theorem 1, the restricted $f_{1}$ must have Lipschitz constant equal to $\sqrt{2}$, and agree with the restricted $p_{1}$ up to isometries of domain and range.

In other words, $f_{1}: V_{v} \cong S^{3}(\sqrt{2}) \rightarrow S^{2}$ is a Hopf projection, scaled up by $\sqrt{2}$.

Claim The image $f\left(V_{v}\right)$ is a horizontal 2-sphere $S^{2} \times v^{\prime}$ in $S^{2} \times S^{2}$.

Proof of claim We already know that $f_{1}: V_{v} \cong S^{3}(\sqrt{2}) \rightarrow S^{2}$ is a Hopf projection, whose fibers are great circles of radius $\sqrt{2}$.

In particular, the map $f_{1}$ is smooth.

In the tangent space to $V_{v}$ at the point $(x, x v)$, let $F_{x}$ denote the tangent line to the great circle fiber of $f_{1}$, and $G_{x}$ the orthogonal 2-plane.

The differential of $f_{1}$ at this point takes $G_{x}$ conformally to the tangent 2-plane to $S^{2}$ at the image point, stretching lengths by $\sqrt{2}$.

In particular, any smooth curve in $V_{v}$ everywhere tangent to $G_{x}$ is taken by $f_{1}$ conformally to a curve in $S^{2}$, stretching lengths by $\sqrt{2}$.

Since this is the maximum stretch allowed the map $f: V_{v} \rightarrow G_{2} \mathbb{R}^{4}=S^{2} \times S^{2}$, that same curve in $V_{v}$ must be taken by $f$ to a horizontal curve in $S^{2} \times S^{2}$.

But any two Hopf fibers can be connected by a smooth curve in $S^{3}$ which is everywhere orthogonal to the fibers its passes through, and hence $f$ must take all of $V_{v}$ to a single horizontal 2-sphere $S^{2} \times v^{\prime}$, verifying the claim.

And since $f_{1}: V_{v} \rightarrow S^{2}$ is a Hopf fibration, so also is $f: V_{v} \rightarrow S^{2} \times v^{\prime}$, where it is perfectly possible that $v^{\prime} \neq v$. 
We have thus gained some control over the nature of $f$ : on each $V_{v}$ the map $f$ must be a Hopf projection, with image a horizontal 2-sphere in $S^{2} \times S^{2}$.

The fibers of $f$ must therefore be great circles in $V_{v} \cong S^{3}(\sqrt{2})$, but we don't yet know that they coincide with the Stiefel fibers.

Now repeat all of the above with orthogonal complex structures on $\mathbb{R}^{4}$ given by left multiplication by a purely imaginary unit quaternion $u$, and learn that the image $f\left({ }_{u} V\right)$ is a vertical 2-sphere $u^{\prime} \times S^{2}$ in $S^{2} \times S^{2}$, where again it is possible that $u^{\prime} \neq u$.

The two images $u^{\prime} \times S^{2}$ and $S^{2} \times v^{\prime}$ intersect in the single point $\left(u^{\prime}, v^{\prime}\right)$.

Since the inverse image of an intersection is the intersection of the inverse images, the portion of $f^{-1}\left(u^{\prime}, v^{\prime}\right)$ within ${ }_{u} V \cup V_{v}$ must be ${ }_{u} V \cap V_{v}$, which is a Stiefel fiber.

It follows in this way that the map $f$ must collapse each Stiefel fiber in $V_{2} \mathbb{R}^{4}$ to a single point in $G_{2} \mathbb{R}^{4}$, although we do not yet know that distinct Stiefel fibers are sent to distinct points by $f$.

Since $f: V_{2} \mathbb{R}^{4} \rightarrow G_{2} \mathbb{R}^{4}$ collapses each Stiefel fiber to a point, it induces a map

$$
\bar{f}: G_{2} \mathbb{R}^{4}=S^{2} \times S^{2} \rightarrow G_{2} \mathbb{R}^{4}=S^{2} \times S^{2},
$$

with Lipschitz constant less than or equal to 1.

From the above discussion, we see that $\bar{f}$ takes the horizontal 2 -sphere $S^{2} \times v$ to the horizontal 2-sphere $S^{2} \times v^{\prime}$, and the vertical 2-sphere $u \times S^{2}$ to the vertical 2-sphere $u^{\prime} \times S^{2}$, in each case with Lipschitz constant less than or equal to 1 .

Since by hypothesis the map $f$ is homotopic to the Stiefel projection $p$, these horizontalto-horizontal and vertical-to-vertical maps of 2-spheres are all homotopic to the identity.

But a map from $S^{2}$ to $S^{2}$ which is homotopic to the identity and has Lipschitz constant less than or equal to 1 must be an orientation-preserving isometry.

It follows that

$$
\bar{f}(u, v)=\left(u^{\prime}, v^{\prime}\right)=(g(u), h(v)),
$$

where $g$ and $h$ are orientation-preserving isometries of $S^{2}$.

Hence $f$ differs from the Stiefel projection $p$ by an isometry $(g, h)$ of their common range $G_{2} \mathbb{R}^{4}$, completing the proof of Theorem 4 . 


\section{References}

[1] V Borrelli, F Brito, O Gil-Medrano, The infimum of the energy of unit vector fields on odd-dimensional spheres, Ann. Global Anal. Geom. 23 (2003) 129-140 MR1961372

[2] V Borrelli, O Gil-Medrano, A critical radius for unit Hopf vector fields on spheres, Math. Ann. 334 (2006) 731-751 MR2209254

[3] R Bott, L W Tu, Differential forms in algebraic topology, Graduate Texts in Mathematics 82, Springer, New York (1982) MR658304

[4] F G B Brito, Total bending of flows with mean curvature correction, Differential Geom. Appl. 12 (2000) 157-163 MR1758847

[5] F G B Brito, P Walczak, On the energy of unit vector fields with isolated singularities, Ann. Polon. Math. 73 (2000) 269-274 MR1785691

[6] J Eells, L Lemaire, A report on harmonic maps, Bull. London Math. Soc. 10 (1978) 1-68 MR495450

[7] R H Escobales, Jr, Riemannian submersions with totally geodesic fibers, J. Differential Geom. 10 (1975) 253-276 MR0370423

[8] F B Fuller, Harmonic mappings, Proc. Nat. Acad. Sci. U. S. A. 40 (1954) 987-991 MR0064453

[9] O Gil-Medrano, Relationship between volume and energy of vector fields, Differential Geom. Appl. 15 (2001) 137-152 MR1857559

[10] O Gil-Medrano, Volume and energy of vector fields on spheres. A survey, from: "Differential geometry, Valencia, 2001”, (O Gil-Medrano, V Miquel, editors), World Sci. Publ., River Edge, NJ (2002) 167-178 MR1922046

[11] O Gil-Medrano, Unit vector fields that are critical points of the volume and of the energy: characterization and examples, from: "Complex, contact and symmetric manifolds", (O Kowalski, E Musso, editors), Progr. Math. 234, Birkhäuser, Boston, MA (2005) 165-186 MR2105148

[12] O Gil-Medrano, E Llinares-Fuster, Minimal unit vector fields, Tohoku Math. J. 54 (2002) 71-84 MR1878928

[13] H Gluck, F W Warner, Great circle fibrations of the three-sphere, Duke Math. J. 50 (1983) 107-132 MR700132

[14] H Gluck, F Warner, W Ziller, The geometry of the Hopf fibrations, Enseign. Math. 32 (1986) 173-198 MR874686

[15] H Gluck, F Warner, W Ziller, Fibrations of spheres by parallel great spheres and Berger's rigidity theorem, Ann. Global Anal. Geom. 5 (1987) 53-82 MR933907

[16] H Gluck, W Ziller, On the volume of a unit vector field on the three-sphere, Comment. Math. Helv. 61 (1986) 177-192 MR856085 
[17] D Gromoll, K Grove, One-dimensional metric foliations in constant curvature spaces, from: "Differential geometry and complex analysis", (I Chavel, H M Farkas, editors), Springer, Berlin (1985) 165-168 MR780042

[18] D Gromoll, K Grove, The low-dimensional metric foliations of Euclidean spheres, J. Differential Geom. 28 (1988) 143-156 MR950559

[19] D-S Han, J-W Yim, Unit vector fields on spheres, which are harmonic maps, Math. Z. 227 (1998) 83-92 MR1605381

[20] H Hopf, Über die Abbildungen der dreidimensionalen Sphäre auf die Kugelfä̈che, Math. Ann. 104 (1931) 637-665 MR1512691

[21] H Hopf, Uber die Abbildungen von Sph"aren auf Sph"aren niedrigerer Dimension, Fund. Math. 25 (1935) 427-440

[22] T Ishihara, Harmonic sections of tangent bundles, J. Math. Tokushima Univ. 13 (1979) 23-27 MR563393

[23] D L Johnson, Volumes of flows, Proc. Amer. Math. Soc. 104 (1988) 923-931 MR964875

[24] J J Konderak, On harmonic vector fields, Publ. Mat. 36 (1992) 217-228 MR1179614

[25] O Nouhaud, Applications harmoniques d'une variété riemannienne dans son fibré tangent. Généralisation, C. R. Acad. Sci. Paris Sér. A-B 284 (1977) A815-A818 MR0431035

[26] S L Pedersen, Volumes of vector fields on spheres, Trans. Amer. Math. Soc. 336 (1993) 69-78 MR1079056

[27] A Ranjan, Riemannian submersions of spheres with totally geodesic fibres, Osaka J. Math. 22 (1985) 243-260 MR800969

[28] S Sasaki, On the differential geometry of tangent bundles of Riemannian manifolds, Tôhoku Math. J. 10 (1958) 338-354 MR0112152

[29] S W Wei, Classification of stable currents in the product of spheres, Tamkang J. Math. 42 (2011) 427-440 MR2862346

[30] J H C Whitehead, An expression of Hopf's invariant as an integral, Proc. Nat. Acad. Sci. U. S. A. 33 (1947) 117-123 MR0020255

[31] B Wilking, Index parity of closed geodesics and rigidity of Hopf fibrations, Invent. Math. 144 (2001) 281-295 MR1826371

[32] J A Wolf, Elliptic spaces in Grassmann manifolds, Illinois J. Math. 7 (1963) 447-462 MR0156295

[33] J A Wolf, Geodesic spheres in Grassmann manifolds, Illinois J. Math. 7 (1963) 425-446 MR0156294 
[34] Y-C Wong, Isoclinic n-planes in Euclidean 2n-space, Clifford parallels in elliptic $(2 n-1)-$ space, and the Hurwitz matrix equations, Mem. Amer. Math. Soc. No. 41 (1961) MR0145437

[35] C M Wood, On the energy of a unit vector field, Geom. Dedicata 64 (1997) 319-330 MR1440565

[36] C M Wood, The energy of Hopf vector fields, Manuscripta Math. 101 (2000) 71-88 MR1737225

[37] Y L Xin, Some results on stable harmonic maps, Duke Math. J. 47 (1980) 609-613 MR587168

Department of Mathematics, University of Pennsylvania

David Rittenhouse Laboratory, 209 South 33 Street, Philadelphia, PA 19104-6395, USA

deturck@math.upenn.edu, gluck@math.upenn.edu, peterastorm@gmail.com

Received: 23 May 2012 Revised: 12 October 2012 